\title{
Optical Parametric Amplification Techniques for the Generation of High-Energy Few-Optical-Cycles IR Pulses for Strong Field Applications
}

\author{
Anna G. Ciriolo ${ }^{1}$, Matteo Negro ${ }^{2}$, Michele Devetta ${ }^{2}$, Eugenio Cinquanta ${ }^{1}$, Davide Faccialà ${ }^{1}$, \\ Aditya Pusala ${ }^{1}$, Sandro De Silvestri ${ }^{1,2}$, Salvatore Stagira ${ }^{1,2}$ and Caterina Vozzi ${ }^{2, *}$ \\ 1 Dipartimento di Fisica, Politecnico di Milano, Piazza Leonardo da Vinci, 32, 20133 Milano, Italy; \\ annagabriella.ciriolo@polimi.it (A.G.C.); eugenioluigi.cinquanta@polimi.it (E.C.); davide.facciala@polimi.it \\ (D.F.); Aditya.pusala21@gmail.com (A.P.); \\ sandro.desilvestri@polimi.it) (S.D.S.); salvatore.stagira@polimi.it (S.S.) \\ 2 Istituto di Fotonica e Nanotecnologie (IFN), CNR, Via Cineto Romano, 42, 00156 Roma, Italy; \\ matteo.negro@polimi.it (M.N.); michele.devetta@mail.polimi.it (M.D.) \\ * Correspondence: caterina.vozzi@cnr.it; Tel.: +39-02-2399-6085
}

Academic Editor: Antonella Bogoni

Received: 20 January 2017; Accepted: 1 March 2017; Published: 8 March 2017

\begin{abstract}
Over the last few decades, the investigation of ultrafast phenomena occurring in atoms, molecules and solid-state systems under a strong-field regime of light-matter interaction has attracted great attention. The increasing request for a suitable optical technology is significantly boosting the development of powerful ultrafast laser sources. In this framework, Optical Parametric Amplification (OPA) is currently becoming a leading solution for applications in high-power ultra-broadband light burst generation. The main advantage provided by the OPA scheme consists of the possibility of exploring spectral ranges that are inaccessible by other laser technologies, as the InfraRed (IR) window. In this paper, we will give an overview on recent progress in the development of high-power few-optical-cycle parametric amplifiers in the near-IR and in the mid-IR spectral domain. In particular, the design of the most advanced OPA implementations is provided, containing a discussion on the key technical aspects. In addition, a review on their application to the study of strong-field ultrafast physical processes is reported.
\end{abstract}

Keywords: ultrafast optics; optical parametric amplifiers; high order harmonics; attosecond science

\section{Introduction}

Ultrafast laser technology is rapidly developing under the impulse of exciting perspectives of applications. In particular, time-resolved spectroscopy of physical systems is driving progresses in ultrashort light pulse generation, aiming at understanding and controlling nuclear and electronic dynamics on an extremely short time scale, down to the attosecond domain.

In the 1990s, mode-locking-based solid-state laser systems were introduced, soon becoming the dominant solution for ultrafast applications, by enabling ultra-short pulse duration on the few-femtosecond scale [1]. The interest in investigating the ultrafast light-matter interaction regime under strong-field perturbations boosted the power scaling of solid-state sources. In this framework, the Chirped Pulse Amplification (CPA) [2] scheme was proposed, which enabled single pulse energy enhancement up to three orders of magnitude, thus attaining the mJ-level.

Since solid-state sources provide pulses centered at a well-defined wavelength, typically in the visible part of the spectrum, a great effort has been dedicated to the development of tunable sources. In this sense, Optical Parametric Amplification (OPA) provides a practicable strategy for intense 
few-cycle laser pulse generation, even in those spectral regions that are not supported by amplified solid-state laser sources [3].

In this paper, the attention is focused on describing current progress in high-energy few-cycle parametric sources tunable in the IR spectral window beyond $800 \mathrm{~nm}$. The structure of the paper is presented below. In Section 2, a brief overview of the operating principles of parametric amplification is provided. Section 3 is devoted to the description of the most advanced OPA implementations for IR pulse generation. Before illustrating in detail practical implementations, a preliminary discussion on the main technological and operational issues underlying the development of these ultrafast optical sources is provided. Finally, applications to the study of strong-field phenomena are reported in Section 4.

\section{Overview on the Optical Parametric Amplification Process}

The basic working principles of parametric amplifiers are briefly described here. For a comprehensive overview, the reader is addressed to [4].

OPA is a well-known nonlinear optical process occurring in second-order non-linear crystals and involving energy transfer among beams at different frequencies $[5,6]$. A simple sketch of the process is presented in Figure 1: energy is transferred from a high intensity beam (called the pump beam, at frequency $\omega_{p}$ ) to a lower intensity beam (called the signal beam, at frequency $\omega_{s}$ ), thus resulting in an amplification of the signal. During the pump-beam interaction, a third beam is generated (called the idler beam, at frequency $\omega_{i}$ ) in order to fulfill energy conservation. For a pump frequency higher than the signal and idler frequencies, the conversion rule is expressed as $\hbar \omega_{p}=\hbar \omega_{s}+\hbar \omega_{i}$. In the case of OPA systems working in the IR, extremely powerful laser sources in the visible/near-IR spectral range can be used for pumping a parametric process of downconversion into longer wavelengths.

\section{a}

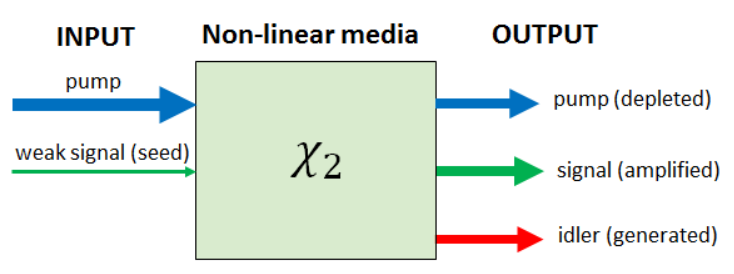

b

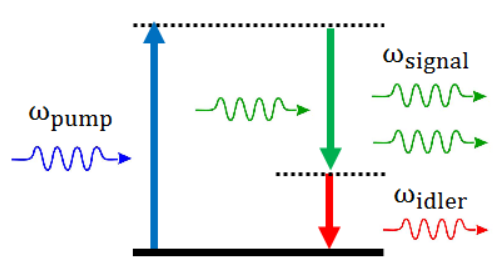

Figure 1. (a) Power flow from the pump beam to the signal beam in optical parametric amplification; (b) the same optical parametric amplification process can be viewed as stimulated emission of signal photons from a virtual level excited by the pump.

Parametric amplification takes place all along the non-linear media, and it is strongly affected by propagation. Indeed, pump-to-signal/idler photon conversion occurs locally in the material with the local phase of the three fields being coupled by a precise relationship: $\phi_{p}=\phi_{s}+\phi_{i}$. Then, the boosted signal and idler beams propagate inside the non-linear crystals. During propagation, they overlap with other photon contributions arising from different sites, resulting in a spatial interference. To observe a macroscopic effect of amplification, a constructive interference regime has to be achieved. Constructive interference can be achieved by properly tuning the phase velocities of the propagating waves. In particular, the following condition, known as the phase-matching condition, must be satisfied for the pump, signal and idler wavevectors (respectively, $\left.\overrightarrow{k_{p}}, \overrightarrow{k_{s}}, \overrightarrow{k_{i}}\right): \overrightarrow{k_{p}}=\overrightarrow{k_{s}}+\overrightarrow{k_{i}}$. In order to fulfill the phase-matching condition, birefringent non-linear media, with uniaxial or biaxial optical properties, are exploited. In this case, the phase velocity of each beam inside the crystal is defined according to the orientation of the polarization and to the direction of propagation.

In general, beams with different polarizations and different propagation directions are used. Polarization and propagation direction are the two main degrees of freedom available to the users for 
optimizing the OPA systems. In particular, by properly choosing these two parameters, phase-matching conditions can be simultaneously fulfilled for a very broad range of signal frequencies, thus resulting in a broadband amplification. In this sense, two interaction types can be distinguished depending on the polarization states of the three incoming waves: Type-I (signal and idler propagating with the same polarization, either ordinary or extraordinary) and Type-II (signal and idler propagating with orthogonal polarization, either ordinary or extraordinary). Type-I phase-matching provides signal amplification on a broad spectral region. However, this interaction type is typically associated with large spectral dispersion. On the other hand, Type-II phase-matching results in a narrower phase-matching band, suitable for delivering non-dispersed signal pulses. Concerning the propagation direction, the non-collinear pump-signal configuration was proven to ensure suitable phase-matching on an extended signal band, thus enabling a broadband parametric amplification.

Broadband OPAs are ideal candidates for amplifying ultrashort pulses. When ultrashort pulses are used as an input for amplification, wave packet effects have to be mentioned. First, for energy transfer to occur from the pump to the signal/idler, spatial and temporal superposition of the three pulses is required. However, this superposition gets gradually lost along the propagation direction because of the group velocity mismatch. Thus, OPA systems are designed for providing multiple amplification stages, to compensate for this temporal walk-off dynamics. Moreover, for a fixed pump and signal propagation direction, the idler propagation direction is deduced by the wavevector equations. In a broad bandwidth amplification configuration, a number of spectral components of the signal beams must be phase-matched. The corresponding idler components arise so as to match both energy and wavevector equations. As a result, the idler beam is affected by spatial chirp.

It is worth mentioning here that OPA originally arises as an alternative to amplification systems based on population inversion in laser active media. With respect to the active media systems, OPA provides a number of advantages, which are here briefly discussed $[7,8]$.

Unlike stimulated emission in active media, parametric amplification is not a resonant process, and it does not involve any real energy levels, the non-linear materials acting exclusively as an intermediate during the three-wave interaction. As a consequence, OPA systems can potentially work at any wavelength, even in those spectral regions that are not supported by lasing media. Moreover, by properly varying the phase-matching conditions, different spectral components can be selected for amplification. OPAs thus provide highly tunable sources, the region of tunability being subjected to the above-mentioned energy conservation rule. The main restriction limiting this amplification band is set by the transparency range of the non-linear crystal. Indeed, the energy exchange process among the pump, signal and idler waves is boosted by their mutual interaction; consequently, for the non-linear interaction to occur efficiently, transparency is required at the three frequencies involved. Transparency at the working wavelengths prevents thermal effects, which are instead particularly critical in amplification systems based on stimulated emission, strongly limiting power scalability. As a major advantage, in OPAs, the gain bandwidth is dictated by the possibility to satisfy the phase-matching condition over a broad spectral range. First of all, restrictions due to the line width of electronic or vibrational lasing transitions are avoided. Moreover geometrical degrees of freedom are available in birefringent media for optimizing the parametric amplification gain bandwidth, involving the propagation direction and the polarization of the interacting waves. A number of OPA architectures have been introduced for achieving broadband phase-matching. In this sense, ultra-broadband OPAs have been experimentally demonstrated, delivering pulses with durations down to the single-cycle limit from the visible to the mid-IR spectral region [9].

Actually, parametric amplification systems are approaching the intensity levels provided by the most advanced amplified laser sources [10]. Moreover, they were established over the years as a powerful technology for applications related to ultrafast spectroscopy and for strong field applications [11]. 


\section{High-Energy Few-Optical Cycle Parametric Sources in the IR Spectral Region}

In order to achieve high energy pulses in the IR as a result of a parametric amplification, high-energy pumping pulses are required. Indeed, the efficiency of the pump-to-signal energy conversion fundamentally depends on the system design and usually stands around a few tens of percent [8]. For this reason, mJ-level OPAs are mainly pumped by amplified femtosecond Ti:sapphire (Ti:Sa) lasers systems providing pulses at $800 \mathrm{~nm}$ with terawatt peak powers [12]. Ti:Sa has been established as the leading technology in OPA systems, both for the peak-power achievable and for the unsurpassed broad bandwidth they exhibit, enabling the generation of high-energy pump pulses below 20 fs. Pump pulse duration is a critical issue in parametric systems. For attaining an efficient energy transfer in a few-cycle parametric regime, a duration-matching is beneficial, with the pump pulse duration being on the same scale of the signal pulse duration. Femtosecond Ti:Sa-based OPAs have currently been sided by high-energy narrow-band sources, based on $\mathrm{Nd}$ - and $\mathrm{Yb}$-doped active media, in a new class of parametric amplifiers, namely Optical Parametric Chirped Pulses Amplifiers (OPCPAs). In OPCPAs, restrictions related to the use of femtosecond pump sources are overcome by means of a technique inspired by the Chirped Pulse Amplification (CPA). Specifically, picosecond pump lasers (at $800-1100 \mathrm{~nm}$ ) are used for amplifying temporally pre-dispersed IR signal pulses [13]. Sections 3.1 and 3.2 are devoted to the description of femtosecond-pumped OPAs and picosecond-pumped OPCPA high energy few-cycle IR sources, respectively.

The amplified signal inherits the temporal and spectral properties of the seed pulse. Thus, the possibility to produce IR signal pulses supporting the few-cycle regime relies on the exploitation of the broadband seed generation techniques. Another crucial characteristic of the seed pulse in the few-cycle regime is the Carrier-Envelope Phase (CEP), which is defined as the phase offset between the carrier and the envelope of the pulse. The stabilization of the CEP enables the generation of optical waveforms with a reproducible electric field profile. In the few-cycle regime, a change in the CEP produces a strong variation in the waveform, affecting strongly non-linear optical phenomena, such as multiphoton absorption, above-threshold ionization and high-harmonic generation. CEP control is a crucial pre-requisite for the generation of isolated attosecond pulses.

The main techniques adopted for generating a broadband seed in the IR spectral region are Parametric SuperFluorescence (PSF), White Light Generation (WLG) and Difference Frequency Generation (DFG). PSF consists of a spontaneous emission process, such that the seed is generated in a second-order non-linear crystal from vacuum fluctuations or quantum noise, under the effect of a pump pulse radiation with a relatively low intensity [14]. A broadband seed tunable from the near- to the mid-IR range depending on the non-linear media is generated, with the IR emission being subject to phase-matching conditions with the pump beam [15]. Since the process is initiated by noise, large random pulse-to-pulse energy fluctuations occur. However, this is generally a minor issue in PSF-based OPAs; indeed, those fluctuations undergo attenuation as a result of the subsequent parametric amplification. Moreover, due to the stochastic nature of emission, a timing jitter between pump and seed exists, making the synchronization of pulses in the OPA a fundamental issue. As a major drawback of PSF, the CEP relation between the pump and the PSF pulses is completely lost [16], and seed pulses exhibit a completely random CEP.

Higher pump pulse intensity is required for WLG, but significant advantages can be obtained in terms of shot-to-shot stability and CEP-control. WLG occurs in dielectric media with a third-order non-linearity. It involves spectral broadening of the pumping pulses by means of a combination of three-photons processes as Four Wave Mixing (FWM) and Self-Phase Modulation (SPM) [17]. By filtering the low-frequency components, a seed is obtained extending up to the near-IR. In this process, the CEP value of the driving pulses is preserved [18-20] so that a CEP-stable seed is produced provided that CEP-stable pump pulses are used. On the other hand, if the pump and seed have a well-defined phase relationship between them, a CEP-stable idler beam is obtained under parametric amplifications by phase differencing between the two contributing pulses. 
Finally, DFG occurs in second-order non-linear crystals excited by highly intense ultra-broadband pump pulses. It can be described as a two-photon process in which frequency differencing takes place, so that new components are generated in the long wavelength domain. As opposed to PSF and WLG, DFG potentially has the advantage of generating CEP-locked pulses in the near-IR and mid-IR, starting from non-CEP-stabilized driving pulses [21,22]. Indeed, intrapulse DFG is associated with a phase differencing operation between the two mixing frequencies, thus providing insensitivity to pump CEP fluctuations [23].

In the following sections, a number of OPA implementations will be described based on different seed generation approaches, depending on the IR region of interest for amplification and on the CEP stabilization strategy.

As a technological issue, the development of the high-energy ultrafast parametric source in the IR is strongly affected by the availability of suitable Non-Linear Optical (NLO) crystals. Indeed, NLO crystals have to be properly selected according to a number of requirements. In particular, crystals exhibiting a large second-order nonlinear coefficient and a broadband birefringent phase-matching over the near-IR and mid-IR spectral windows are required for an efficient parametric amplification. Moreover, in order to support high pumping power, crystals with high thermal conductivity and low absorption losses at the pump-pulse wavelengths have to be used. Importantly, transparency in the signal and idler spectral domain has to be fulfilled.

Most OPA and OPCPA systems in the near-IR rely on $\beta-\mathrm{BaB}_{2} \mathrm{O}_{4}$ (BBO) as a nonlinear crystal. A number of other materials, belonging to the oxide-family may be cited as ideal candidates for near-IR parametric amplification, as $\mathrm{KH}_{2} \mathrm{PO}_{4}(\mathrm{KDP}), \mathrm{KTiOAsO}_{4}(\mathrm{KTA}), \mathrm{KTiOPO}_{4}(\mathrm{KTP}), \mathrm{LiB}_{3} \mathrm{O}_{5}$ (LBO) and $\mathrm{LiNbO}_{3}$. However, so far, their exploitation was fundamentally limited by the difficulties associated with the fabrication process, preventing the growth of large-size crystals with a good optical quality. By using the above-mentioned NLO media, tunability can be achieved up to wavelengths on the order of a few $\mu \mathrm{m}$ (shorter than $\sim 3 \mu \mathrm{m}$ ) by the onset of IR absorption. Parametric amplification in the IR domain is at the cutting edge of parametric source technology. When the DFG process generates a seed pulse in the terahertz $(\mathrm{THz})$ domain, the process is called optical rectification, and it is widely benefiting from a new generation of non-oxide materials capable of providing optical transparency up to tens of $\mu \mathrm{m}$, among which $\mathrm{AgGaSe}_{2}$ (AGSe), ZnGeP2 (ZGP), GaSe, GaP and ZnTe. A full list of NLO materials is provided in [24] for applications in the near-IR and in [25-27] for applications in the mid-IR.

An overview on the main technological solutions applied in the currently available high-energy few-cycle parametric amplifiers can be inferred from the following paragraphs, where a detailed description of the different experimental setups is reported.

\subsection{Available Sources Based on the OPA Design}

In this section, we report a description of some of the OPA systems currently available and capable of providing high energy few-cycle CEP-stabilized IR pulses.

\subsubsection{Millijoule-Level IR Parametric Sources}

A parametric amplifier in the near-IR spectral region was demonstrated in 2006 by Vozzi et al. [23], providing $200-\mu \mathrm{J}, 15$-fs CEP-stable pulses at $1.5 \mu \mathrm{m}$. Using a similar design, the system was subsequently scaled up to the mJ level $[28,29]$, making it suitable for experiments in the strong-field regime. A detailed description of the mJ-energy source is reported below.

This source consists of broadband near-IR OPA, centered around $1.5 \mu \mathrm{m}$, providing millijoule-level pulses, which are passively stabilized by means of a DFG seeding process. A schematic view of its architecture is shown in Figure 2a. 
(a)

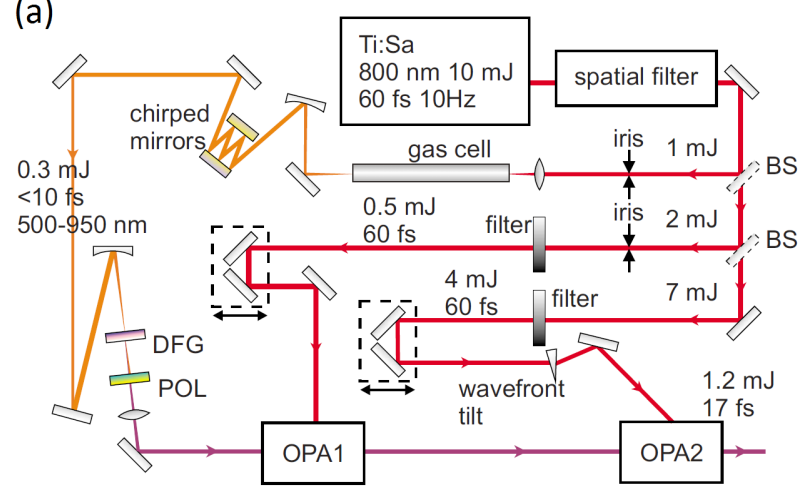

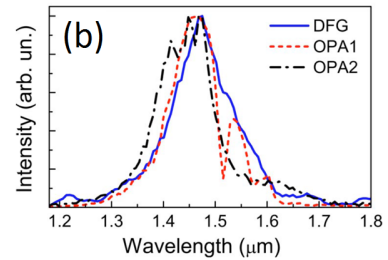

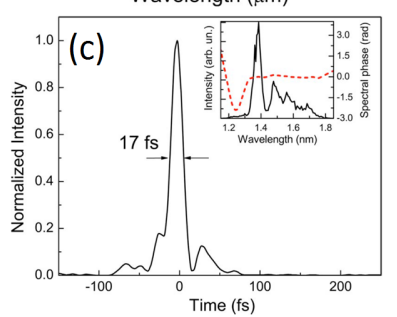

Figure 2. (a) Scheme of the Optical Parametric Amplification (OPA) setup for the generation of highenergy Carrier-Envelope Phase (CEP)-stable near-IR pulses: BS, Beam Splitter; Difference Frequency Generation (DFG), $400 \mu \mathrm{m}$-thick BBO crystal; POL, Polarizer; filter, neutral density filter for controlling intensity of the pump beam; (b) Spectra of the DFG pulses (solid curve) and of the pulses after the first (dashed curve) and second (dashed-dotted curve) OPA stage in a Type-II phase-matching configuration; (c) Temporal intensity profile corresponding to a pulse duration of $17 \mathrm{fs}$. The inset shows the spectrum of the amplified IR pulses (solid curve) and the retrieved spectral phase (dashed curve). Adapted with permission from [28], Copyright OSA Publishing, 2007. Adapted with permission from [29], Copyright OSA Publishing, 2008.

A Ti:sapphire (Ti:Sa) laser system, providing $60-\mathrm{fs}$ pulses at $\lambda=800 \mathrm{~nm}$ with an energy of $10 \mathrm{~mJ}$ and a repetition rate of $10 \mathrm{~Hz}$, is used for driving the parametric process. Its energy is split into three portions, one for generating an IR seed and two for pumping the two amplification stages.

A phase-stable seed is generated through DFG among different spectral components of a spectrally-broadened laser pulse. Specifically, a small fraction of the driving laser beam $(1 \mathrm{~mJ})$ is focused through an iris in a krypton-filled gas cell. Here, spectral broadening occurs by filamentation [30]. The out-coming broadband pulses have an energy around $0.3 \mathrm{~mJ}$ and are compressed by means of chirped mirrors down to a duration of around $10 \mathrm{fs}$. These pulses are then focused into a $200 \mu \mathrm{m}$-thick $\mathrm{BBO}$ crystal, where the DFG process takes place. As a result, a nJ-level CEP-stable IR seed is generated, which is subsequently boosted in energy by optical parametric amplification.

Parametric amplification takes place in two stages. The first OPA stage consists of a 2-mm BBO crystal pumped by 2-mJ pulses, while the second one consists of a 3-mm BBO crystal pumped by $7-\mathrm{mJ}$ pulses. In the second amplification stage, a small prism was inserted in the pump pathway in order to tilt the pulse front of $2^{\circ}$ so as to match it with the pulse front of the seed beam [31]; as a result, the energy transfer from the pump to the seed is maximized. A slightly non-collinear Type-II phase-matching configuration is exploited in both stages for achieving few-cycle near-IR pulses. A spectral characterization of the system is reported in Figure $2 b$, showing the DFG spectrum (solid curve), as well as the IR spectra after the first (dashed curve) and the second (dashed-dotted curve) parametric amplification stages. The energy of the amplified DFG pulse after the second stage of amplification is $1.2 \mathrm{~mJ}$, and its duration is around $17 \mathrm{fs}$, corresponding to 3.5 optical cycles (Figure 2c). As far as the energy stability, the measured peak-to-peak root-mean-squared (rms) fluctuation is better than $4 \%$. Moreover the CEP stability of the out-coming IR pulses was characterized by means of an $f-2 f$ optical system. As a result, an rms fluctuation of the CEP of $220 \mathrm{mrad}$ was measured (Figure 3), which proved the passive stabilization mechanism to provide excellent performances in shot-to-shot stability.

It is worth mentioning that, thanks to the low dispersion of BBO in the spectral region of interest, the IR pulses provided by this system are nearly transform-limited and do not require any further compression. 


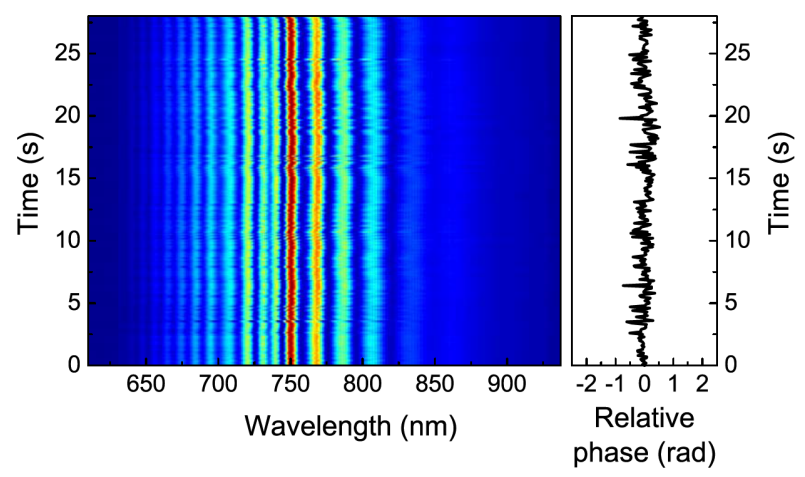

Figure 3. A sequence of interferograms acquired with an $f$-2f interferometer over $28 \mathrm{~s}$ is reported. In the right panel, the CEP fluctuations are shown corresponding to a rms value of $220 \mathrm{mrad}$. Adapted with permission from [29], Copyright OSA Publishing, 2008.

\subsubsection{Sub-Two-Cycle IR Sources Based on Narrowband Parametric Amplification System}

In the OPA system described above, a very powerful Ti:Sa laser source is used for achieving broadband IR pulse with an energy on the millijoule-level. On the other hand, high-power lasers suffer for strict limitations on the repetition rate. In fact, in order to avoid damage and thermal degradation effects inside the laser active media, a low repetition rate was used.

A different scheme is now presented for producing few-cycle IR pulses that is based on a narrowband parametric amplification stage followed by a spectral broadening stage. In this scheme, higher repetition-rate laser sources are used, even if some price is paid in terms of energy.

The pulses originating from a narrow-band parametric amplifier are spectrally broadened, by exploiting a non-linear process occurring in noble gas atoms, namely the Self-Phase Modulation (SPM) process.

The possibility to combine a narrow-band OPA and a compression stage based on SPM strongly relies on the capability to produce IR femtosecond pulses with an energy above the threshold required for efficiently driving non-linear processes in noble gases. In this sense, a first implementation was proposed by Hauri et al. in 2007 [32]. In their setup, a multi-cycle CEP-stabilized IR OPA pumped by a 1-kHz Ti:Sa laser system is used, producing pulses at $2 \mu \mathrm{m}$ with an energy around $500 \mu \mathrm{J}$ and a duration above $50 \mathrm{fs}$. SPM in a plasma filament is then used for broadening the pulses spectrum, thus approaching the few-optical-cycles regime. CEP stability is preserved during filamentation, and the residual rms CEP drift of the output pulses is smaller than $0.1 \mathrm{rad}$. This scheme thus allows for the generation of CEP-stable $270 \mu \mathrm{J}$ pulses, with a measured FWHM of $17 \mathrm{fs}$.

An alternative approach for generating even shorter pulses in the sub-two-cycle regime was reported in 2011 by Schmidt et al. [33,34] based on spectral broadening in a Hollow Core Fiber (HCF). In Figure 4, their experimental setup is shown. It consists of a high energy OPA combined with a pulse broadening and compression stage. The OPA is pumped by a non-CEP-stabilized $1-\mathrm{kHz}$ Ti:Sa laser delivering $3.8 \mathrm{~mJ}$, 35-fs pulses at a wavelength of $800 \mathrm{~nm}$. The laser power is split into four branches, one used for the generation of a seed and the remaining ones used for pumping three amplification stages.

A broadband seed is produced by focusing the radiation into a sapphire plate where white-light generation takes place. As already mentioned, the white-light generation process preserves the CEP fluctuations of the original laser pulses. Thus, a non-CEP stable seed is generated in the near-IR spectral range, which undergoes parametric amplification into a Type-II BBO crystal (BBO 1). Here, the signal is amplified, and an idler is generated at $1.8 \mu \mathrm{m}$. Since both pump and seed share the same CEP fluctuations, the idler is CEP-stable, its phase resulting from the difference between pump and seed phases. In this first parametric stage, a collinear configuration is used, in order to avoid the idler from being affected by spatial chirp. The $1.8 \mu \mathrm{m}$-idler is selected by means of a dichroic 
mirror and amplified in the two subsequent parametric amplification stages (BBO 2, BBO 3), which are implemented using a slightly non-collinear Type-II phase-matching configurations. After BBO 3 , near-IR pulses at $1.8 \mu \mathrm{m}$ with an energy of $650 \mu \mathrm{J}$ and an almost transform-limited duration of $35 \mathrm{fs}$ are available. In order to reduce pulse duration down to the few-cycles scale, these pulses are focused into an argon-filled HCF using a 1-m focusing optic. Here, SPM takes place, leading to a significant spectral broadening. The out-coming pulses have an energy of $240 \mu \mathrm{J}$; the pulse spectrum is very broad extending from $1.15 \mu \mathrm{m}$ to $2.15 \mu \mathrm{m}$, but a residual chirp is present, which is compensated using a plate made up of fused silica. After compression, nearly transform-limited durations of $9.5 \mathrm{fs}$ are obtained, corresponding to 1.6 optical cycles.

(a)

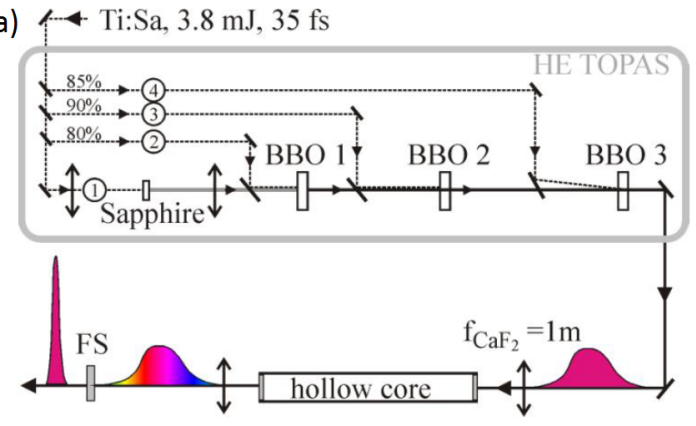

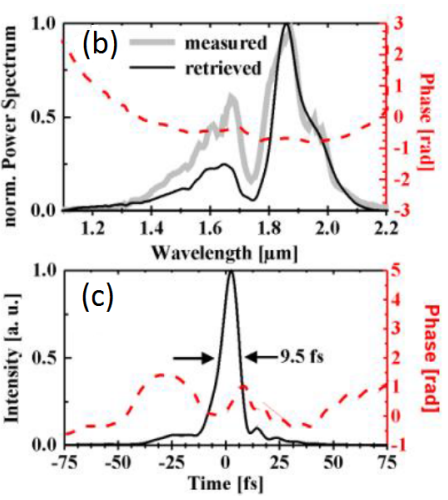

Figure 4. (a) Scheme of the OPA setup for the generation of sub-two-cycle CEP-stable near-IR pulses; (b) results of the IR pulse temporal characterization: measured (gray) and retrieved spectral intensity (black) and phase (dashed red); (c) temporal intensity profile corresponding to a pulse duration of 9.5 fs. FS, Fused Silica. Adapted with permission from [33], Copyright OSA Publishing, 2011.

The energy fluctuation stands around 3.5\% after the OPA, around $6 \%$ after the HCF. As far as the CEP stability is concerned, a 350-mrad rms fluctuation is measured.

If compared to the OPA system from [28] described in the previous subsection, the CEP fluctuations provided by the HCF-based setup are larger. This is mainly due to the fact that an additional source of jitter is introduced in [35], arising from a strong coupling effect in the HCF between pulse intensity and CEP fluctuations. As a result of this coupling effect, a slight variation in the intensity of the radiation entering the fiber can considerably affect the CEP stability. The HCF-based approach is thus very sensitive to pointing instability, as well as intensity instability after the OPA, which are anyway unavoidable due to the non-linear nature of the parametric amplification process. Feedback loop systems can however be installed for achieving better performances in terms of stability.

Definitely, the sources based on HCF, as well as filamentation have the main advantage of providing more degrees of freedom to the users (type of gas, pressure, propagation distance) for achieving an optimum control on the spectral bandwidth of the output radiation, thus allowing for sub-two-cycle pulse generation. However, some limitations are set in the pulses energy, standing at the hundred $\mu$-level.

\subsubsection{High-Energy Tunable IR Parametric Source}

For OPA systems based on a design as the one described in the previous sections, the possibility for achieving energy scaling is fundamentally connected to the advances in the front-end laser technology. However, in modern Ti:Sa laser systems, the energy scalability competes with the repetition rate, making the development of high-energy high-repetition rate sources a significant technical issue.

Tunability is a key feature of OPA systems. In order to obtain tunable ultrashort pulses, a broadband seed must be used. However, achieving tunability in an ultrashort pulse duration regime 
is a challenging task, with the spectral dispersion of the signal pulses being the main limitation. Since the spectral phase is extremely sensible to the experimental conditions upon which seed generation, as well as signal amplification occur, an accurate optimization of parameters as pump/seed pulses energy and duration, as well as a suitable seed line design are required.

We present here a novel parametric source recently developed in our laboratories and capable of providing enhanced performances with respect to the OPA systems described in Section 3.1.1, in terms of repetition rate and tunability.

An advanced Ti:Sa front-end laser source is used for pumping a two-stage parametric amplifier, delivering $1 \mathrm{kHz} 1 \mathrm{~mJ} 25$-fs pulses centered at a wavelength that is tunable between $1.3 \mu \mathrm{m}$ and $2 \mu \mathrm{m}$. The OPA scheme is similar to that shown in Figure 2a.

Ti:Sa front-end laser provides $>15 \mathrm{~mJ} 25 \mathrm{fs} 800-\mathrm{nm}$ pulses at a repetition rate of $1 \mathrm{kHz}$. An $\sim 7-\mathrm{mJ}$ portion of the pulse energy is used for driving the parametric amplifier. In particular, $\sim 330 \mu \mathrm{J}$ are sent into the seed generation line. Here, a $\mathrm{Kr}$-filled HCF is used for spectral broadening and pulse compression below $10 \mathrm{fs}$. The output pulses have an ultra-broadband spectrum, from $550 \mathrm{~nm}$ to $1 \mu \mathrm{m}$, nearly approaching an octave extension. DFG is thus performed into a $3 \mathrm{~mm}$-thick BBO crystal. A DFG spectrum is obtained extending from $1.3 \mu \mathrm{m}$ to $2 \mu \mathrm{m}$. CEP-stable DFG pulses are subsequently amplified by means of two amplification stages, where $3 \mathrm{~mm}$-thick BBO crystals are used in a Type-II phase-matching configuration. The first amplification stage is pumped by $\sim 330 \mu \mathrm{J}$ pulses; in the second amplification stage, 6-mJ pump pulses are used. Along the two pump lines, glass windows of proper thickness have been placed for achieving a fine tuning of the pump pulses duration, thus resulting in an optimization of the amplified DFG signal energy and duration. At the output of the OPA system, we obtain ultrashort pulses, tunable according to the seed DFG spectrum. The energy and the duration of the signal pulses slightly depends on the wavelength. The energy varies from $600 \mu \mathrm{J}$ to $1 \mathrm{~mJ}$, the peak value being measured at $1.5 \mu \mathrm{m}$. As far as the duration, temporal characterization measurements at two different wavelengths are shown in Figure $5 \mathrm{a}-\mathrm{d}$. At $1.4 \mu \mathrm{m}$ (Figure $5 \mathrm{a}, \mathrm{b}$ ), a 16-fs pulse duration has been obtained, corresponding to 3.5 optical cycles of the fundamental frequency. At $1.9 \mu \mathrm{m}$ (Figure $5 \mathrm{c}, \mathrm{d}$ ), transform limited pulses have been obtained with a duration of $32 \mathrm{fs}$, corresponding to five optical cycles of the fundamental frequency.
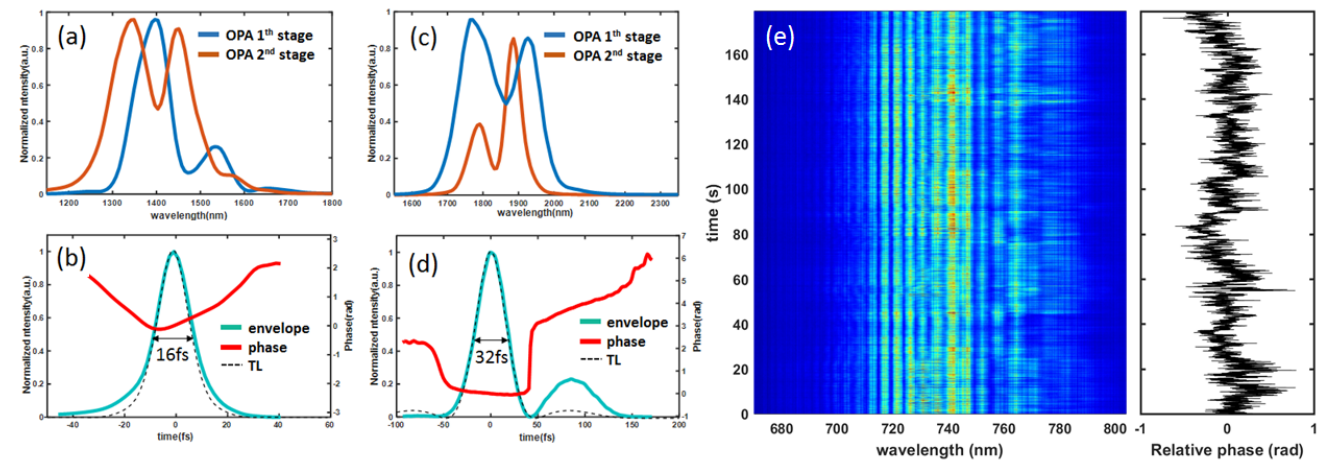

Figure 5. (a) Measured spectral intensity of the signal pulse after the first (blue) and the second (orange) OPA stages tuned at $1.4 \mu \mathrm{m}$; (b) reconstructed temporal intensity profile corresponding to a pulse duration of $16 \mathrm{fs}$; (c) measured spectral intensity of the signal pulse after the first (blue) and the second (orange) OPA stages tuned at $1.9 \mu \mathrm{m}$; (d) reconstructed temporal intensity profile corresponding to a pulse duration of $32 \mathrm{fs}$; (e) a sequence of interferograms acquired with an $f$ - $2 f$ interferometer. In the right panel, the CEP fluctuations are shown corresponding to an rms value of $200 \mathrm{mrad}$.

The CEP stability of the amplified pulses has been quantified by means of $f$ - $2 f$ measurements (see Figure 5e). As a result, an rms value of $200 \mathrm{mrad}$ was measured. An active feedback loop stabilization system is currently being implemented in order to compensate for slow drifts. 


\subsection{Optical Parametric Chirped Pulse Amplification}

In order to scale the OPA energy above the mJ-level, extremely intense pump lasers are required, above the terawatt scale. However, it is technologically challenging to scale the energy of femtosecond pump lasers. On the other hand, energetic picosecond pulses are available based on well-established gain media, such as $\mathrm{Nd}$ - or $\mathrm{Yb}$-doped crystals or fibers that can be exploited for pumping parametric amplifiers.

In this sense, a scheme has been developed known as Optical Parametric Chirped Pulse Amplification (OPCPA) [36,37], which is very similar to the Chirped Pulse Amplification (CPA) occurring in laser gain media. In OPCPA systems, a femtosecond laser system is typically used for generating the seed pulses, while a high peak power picosecond laser is used for generating the pump pulses. Therefore, an energy transfer takes place from ps-pump pulses to fs-signal pulses. In order to optimize energy transfer efficiency, a good temporal overlap is required between these two pulses. Overlap is obtained by first stretching the seed pulse to the picosecond time scale and then, after the amplification step, compressing it back to its nearly Transform-Limited (TL) duration. Besides duration matching, also synchronization of the pump and seed pulses inside the amplification media is required. Synchronization can be achieved either electronically, by using properly-delayed electronic signals for triggering the emission of the pump and seed lasers, or optically, by using a portion of the seed pulse for triggering the pump laser emission. In both cases, pulses synchronization is one of the main issue in OPCPA schemes, contributing to a significant enhancement of the system complexity.

In OPCPAs, parametric amplification is usually implemented in a non-collinear configuration so as to exploit the benefits of a broadband amplification.

Thus, OPCPA systems provide the possibility to amplify extremely broadband optical pulses up to energy levels that are inaccessible by OPA systems because of limitations due to optical damage thresholds. On the other hand, an OPA design presents some advantages with respect to an OPCPA design. In particular, the problem of synchronization is avoided since both pump and seed pulses come from the same laser source. Moreover, there is no need of proper stretching-compression stages on the seed beam. As a result, the experimental setup of an OPA system is considerably simpler than that of an OPCPA system.

\subsubsection{Few-Cycle OPCPA Sources in the Near-IR}

The generation of near-IR millijoule-level few-cycle femtosecond pulses at $1.5 \mu \mathrm{m}$ was reported for the first time by Mücke and coworkers in 2009 [38]. Their OPCPA setup is based on Type-II KTA/KTP crystals pumped by picosecond Nd lasers (Figure 6). The front end of the OPCPA consists of a CEP-stable seeder that provides low-energy IR pulses to be boosted and a Nd:YAG source, providing high-energy pump pulses.

The seeder (see [39] for details) is based on an OPA system driven by a femtosecond source, made up of a Yb:KGW oscillator plus a power amplifier. Specifically, the $130 \mu \mathrm{J}, 250 \mathrm{fs}, 10-\mathrm{kHz}$ pulses at $1.03 \mu \mathrm{m}$ coming out from this source are used to generate a broadband seed by means of white-light generation. The seed undergoes parametric amplification in two OPA stages. Here, idler pulses, CEP-stable, tunable from 1.4 to $1.6 \mu \mathrm{m}$ with an energy of $4 \mu \mathrm{J}$ and a duration sub- $40 \mathrm{fs}$ are generated. These idler pulses are used for seeding the OPCPA.

A portion of the beam produced by the $\mathrm{Yb}: \mathrm{KGW}$ oscillator and centered at $1.064 \mu \mathrm{m}$ is stretched and then delivered to the Nd:YAG system used for pumping. This system consists of a regenerative and a double-pass amplifiers working at a repetition rate of $20 \mathrm{~Hz}$. Amplification of the $1.064 \mu \mathrm{m}$ beam takes place so that $100 \mathrm{~mJ} 60$-fs pulses are generated. 
(a)

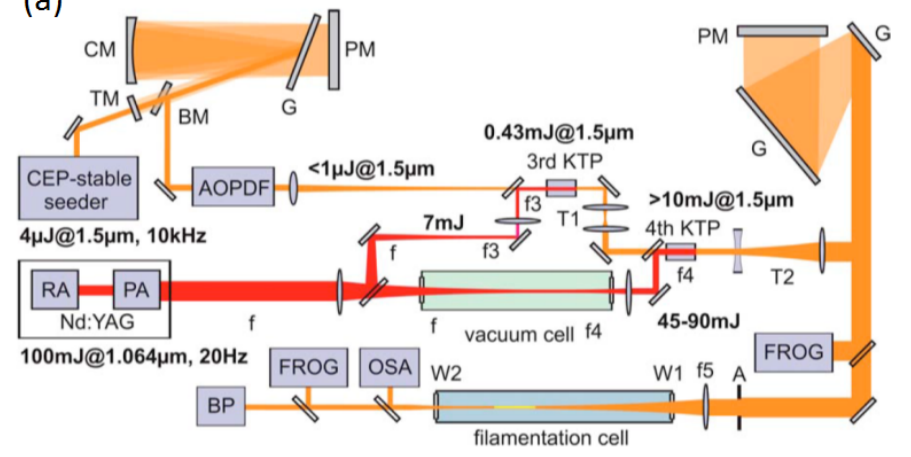

(b)

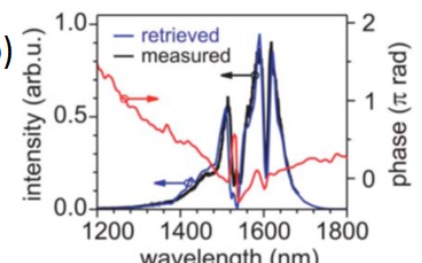

(c)

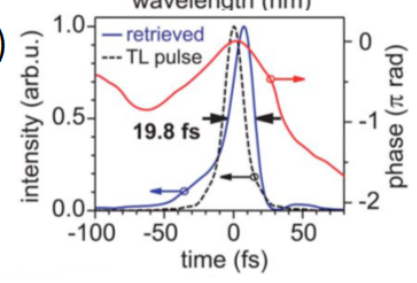

Figure 6. (a) Scheme of the Optical Parametric Chirped Pulse Amplification (OPCPA) setup for near IR pulses amplification. G, reflection Grating; CM, Curved Mirror; PM, Plane Mirror; TM/BM, Top/Bottom Mirror; AOPDF, Acousto-Optic Programmable Dispersive Filter; RA, Regenerative Amplifier; PA, double-pass Post-Amplifier; f, f3, f4, f5, lens Focal lengths; T1, T2, Telescopes; A, Aperture; W1/W2, input/output Windows; BP, Beam Profiler; (b) Measured (black) and retrieved spectral intensity (blue) and phase (red); (c) Temporal intensity profile corresponding to a pulse duration of 19.8 fs. Adapted with permission from [38], Copyright OSA Publishing, 2009.

Synchronization between seed and pump pulses derives from the fact that the beam from the $\mathrm{Yb}: \mathrm{KGW}$ oscillator is used as an input both in the seeder and in the Nd:YAG amplifier.

In order to match pump and seed pulse duration, the seed undergoes temporal stretching inside a grating-based stretcher so that a pulse duration of $40 \mathrm{ps}$ is obtained. An acusto-optic device is used for finely shaping temporal dispersion.

Parametric amplification of the IR seed occurs into two stages, namely Stages 3 and 4. Idler pulses at $\sim 3.4 \mu \mathrm{m}$ are generated.

Both in Stage 3 and in Stage 4, a Type-II phase-matching configuration is chosen. Ten millimeter-thick KTP crystals are used as non-linear media. That is because, unlike borate crystals, KTP is transparent for the idler wavelength in the mid-IR, thus preventing thermal load inside the amplification media and enabling efficient amplification of a signal in the spectral region around $1.5 \mu \mathrm{m}$.

In Stage 3, a 7-mJ pump beam is used, while in Stage 4, the pump energy can be tuned between 45 and $90 \mathrm{~mJ}$. The dimension of the pump beams is changed for matching the dimension of the seed by means of proper telescopes. The high energy in the second pump beam line is delivered to Stage 4 by a vacuum relay imaging system.

As a result of the two parametric amplification steps, CEP-stable pulses at around $1.5 \mu \mathrm{m}$ with an energy up to $12.5 \mathrm{~mJ}$ are generated. The spectral bandwidth of the IR pulses is $80 \mathrm{~nm}$, corresponding to a transform limited duration of $\sim 65$ fs.

To approach the few-cycle regime, spectral broadening by filamentation in a gas cell is performed. In order to prevent additional non-linear effects from affecting the temporal structure of the IR pulse during propagation in the gas, a low input pulse energy is used, $2.2 \mathrm{~mJ}$. With low energy, filamentation in a self-compression regime is achieved. As a result, the IR pulses are compressed down to a 19.8-fs duration, corresponding to four optical cycles of the fundamental frequency. The output energy is $1.5 \mathrm{~mJ}$. By properly optimizing the experimental conditions (beam diameter, focusing length, gas type and pressure, cell length), higher energy can be used as an input for filamentation, and higher output energy $(\sim 5 \mathrm{~mJ})$ can be obtained. However, the energy scalability of this system is low because of energy limitations imposed for attaining a suitable filamentation regime.

Moreover, the extremely high power delivered by the pump source prevents high repetition rate operation regimes. In this sense, an OPCPA system in the near-IR working at a repetition rate of $1 \mathrm{kHz}$ and fully based on Ti:Sa laser technology was recently developed by Ishii and coworkers [40]. 
This system is based on bismuth triborate $\left(\mathrm{BiB}_{3} \mathrm{O}_{6}, \mathrm{BIBO}\right)$ crystals, and it is capable of providing CEP-stabilized sub-two optical cycle pulses at $1.6 \mu \mathrm{m}$ with an energy below the mJ-level $(550 \mu \mathrm{J})$.

A way for increasing amplification gain and bandwidth without the need for a $100 \mathrm{~mJ}$-pump and IR spectral broadening is provided by the use of properly engineered non-linear parametric media: periodically poled non-linear crystals. Periodically poled materials, compared to conventional birefringent crystals, exhibit improved performances, namely a higher bandwidth and an enhanced nonlinear coefficient resulting in higher gain and broadband amplification.

Fuji et al. [41] and $\mathrm{Gu}$ et al. [42] succeeded in developing an OPCPA 2.1- $\mu \mathrm{m}$ system based on $\mathrm{LiNbO}_{3}$ and $\mathrm{LiTaO}_{3}$ periodically-poled crystals. This system produces CEP-stable $740 \mu \mathrm{J}$ pulses at $2.1 \mu \mathrm{m}$ with a duration of $\sim 16 \mathrm{fs}$, corresponding to two optical cycles of the fundamental frequency. This source can in principle be scaled in energy, but the scaling potential of periodically-poled crystals is in turn limited by the difficulty of manufacturing high quality crystals with large apertures.

\subsubsection{Few-Cycle OPCPA Sources in the Mid-IR}

Because of the lack of non-linear media exhibiting transparency in the mid-IR spectral region, parametric amplification of this part of the spectrum has been difficult to achieve. In this sense, a significant advantage offered by periodically-poled crystals is the flexibility of operation in a spectral window, which commonly extends up to the mid-IR range. Titanium compounds, as KTP (Potassium Titanyle Phosphate), KTA (Potassium Titanyle Arsenate) and RTP (Rubidium Titanyle Phosphate), are also suitable for the mid-IR range. Recently, with the introduction of periodically poled crystal technology and with the development of growth techniques for single crystal synthesis, OPCPAs in the mid-IR have been realized.

In $[43,44]$, high repetition rate $(100 \mathrm{kHz})$ microjoule-level hundred-fs pulses at $3 \mu \mathrm{m}$ have been produced by means of OPCPAs systems based on periodically-poled crystals.

The extension to a multi-mJ few-cycle pulse regime was achieved by Andriukaitis and coworkers in 2011 [45]. The scheme of their KTP/KTA-based source is reported in Figure 7. The front end of the system is made up of $\mathrm{Yb}: \mathrm{KGW}$ oscillator, which is used for seeding a 1-mJ $\mathrm{Yb}: \mathrm{Ca} F_{2}$ femtosecond amplifier at $1030 \mathrm{~nm}$ and a 250-mJ Nd:YAG picosecond amplifier at $1064 \mathrm{~nm}$.

(a)

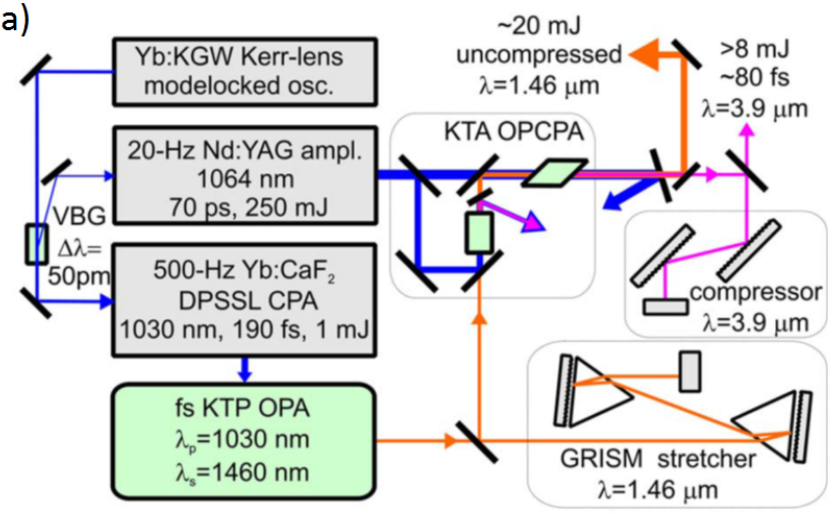

(b)

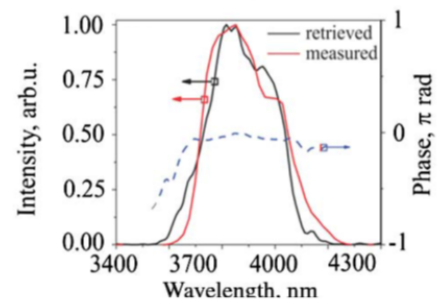

(c)

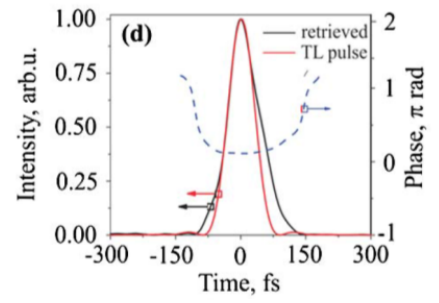

Figure 7. (a) Scheme of the OPCPA set-up for pulses amplification at $3.9 \mu \mathrm{m}$; (b) measured (red) and retrieved spectral intensity (black) and phase (dashed); (c) temporal intensity profile corresponding to a pulse duration of 83 fs. Adapted with permission from [45], Copyright OSA Publishing, 2011.

The $\mathrm{Yb}: \mathrm{Ca}_{2}$ amplifier is used for pumping a white-light-seeded KTP-based OPA at the repetition rate of $0.5 \mathrm{kHz}$. As an output from the OPA, a signal is produced at $1.46 \mu \mathrm{m}$ with an energy of $65 \mu \mathrm{J}$; the phase-stable idler beam at $3.9 \mu \mathrm{m}$ is discarded. The signal pulses are then stretched into a Grating-prism (GRISM) stretcher. 
The Nd:YAG amplifier works at $20 \mathrm{~Hz}$ and is used for pumping two collinear OPCPA stages where $10 \mathrm{~mm}$-long KTA crystals are used as non-linear media. A Type-II phase matching configuration is adopted.

The first OPCPA stage is pumped by $50 \mathrm{~mJ}$, and the $1.46 \mu \mathrm{m}$ seed is amplified to $0.5 \mathrm{~mJ}$.

The second OPCPA stage is pumped by up to $175-\mathrm{mJ}$ pulses. This leads to the generation of uncompressed signal pulses with an energy of $22 \mathrm{~mJ}$ and uncompressed idler pulses at $3.9 \mu \mathrm{m}$, with an energy of $13 \mathrm{~mJ}$. After compression, the $3.9 \mu \mathrm{m}$ idler beam has an energy of $8 \mathrm{~mJ}$. The retrieved idler pulse duration is $83 \mathrm{fs}$, corresponding to less than seven optical cycles of the fundamental frequency. In Figure 7, the idler spectrum and temporal profile after the second OPCPA stage are shown.

Due to the high energy involved in the second OPCPA stage, the KTA crystal has uncoated Brewster cut faces so as to prevent optical damage in the coating. Brewster interfaces lead to angular dispersion in the $1.46-\mu \mathrm{m}$ signal beam entering the crystal. This angular dispersion is transferred to the idler. Thus, the idler beam suffers for a slight divergence, which is however comparable with the corresponding diffraction limit divergence. Moreover, the idler pulses are not CEP stable because they result from the interaction between pump and signal pulses, which are not locked in phase. The system can be modified for providing CEP stable mid-IR pulses by injecting into the OPCPA setup the idler beam coming out from the OPA front end. Indeed, the OPA idler is phase-locked and can be used for seeding the two OPCPA stages by remaining unaffected in phase. The main disadvantage of having the OPA idler as a seed for the OPCPA system consists of the necessity to use suitable mid-IR reflectors throughout the OPCPA setup.

\subsection{Perspectives in OPAs and OPCPAs Development}

An attractive perspective for the future development of compact ultra-broadband tunable parametric OPA and OPCPA in the near-IR and mid-IR lies in the exploitation of fiber-doped laser sources.

Fiber-doped lasers are robust high repetition rate turn-key systems, capable of providing pulses in the near-IR spectral window and characterized by high spatial quality and temporal duration down to the few-tens of fs [46]. The output of these fiber lasers may be used for directly seeding a cascade of OPAs, leading to a substantial reduction of the complexity in the experimental setup. By using a fiber-doped laser system in combination with a powerful ps-solid-state laser system, parametric amplification of the near-IR radiation can be achieved, even above the mJ level. So far, near-IR OPCPA source at the 10-mJ energy-level have been reported $[47,48]$ delivering pulses in the multi-cycle regime ( $\sim 200 \mathrm{fs}$ ) at repetition rate between $10 \mathrm{~Hz}$ and $1 \mathrm{kHz}$.

In recent years, the interest in developing few-cycle long-wavelength sources has fostered research on fiber-based system. That is because the near-IR beam provided by these systems can be used for generating a seed radiation in the mid-IR by DFG process. In $[49,50]$, a dual-output fiber oscillator delivering femtosecond pulses at both 1550 and $1050 \mathrm{~nm}$ is used as a front-end of an OPCPA (Figure 8). In particular, the 3- $\mu \mathrm{m}$ seed pulses for the OPCPA chain are generated by performing DFG between the 1550- and 1050-nm pulses. As a result, a CEP stable mid-IR seed is obtained. The seed is subsequently amplified by means of energy exchange with a $100-\mathrm{kHz}$ ps pump beam in a three-stage OPA. Therefore, few-cycle pulses at $3 \mu \mathrm{m}$ are produced with an energy that is however well below the mJ level $(\sim 5 \mu \mathrm{J})$.

Thus, by integrating fiber-based near-IR sources into OPCPAs, a significant scaling in the setup performances can potentially be attained. In addition, these sources provide the possibility for mid-IR seed light generation directly from a DFG process. This approach is suitable for CEP stability and allows one to overcome limitations discussed for [45], related to the use an idler beam as a mid-IR seed for OPCPAs. On the other hand, the IR pulse energy delivered by these sources is still very low with respect to conventional OPAs and OPCPAs based on solid-state lasers. 


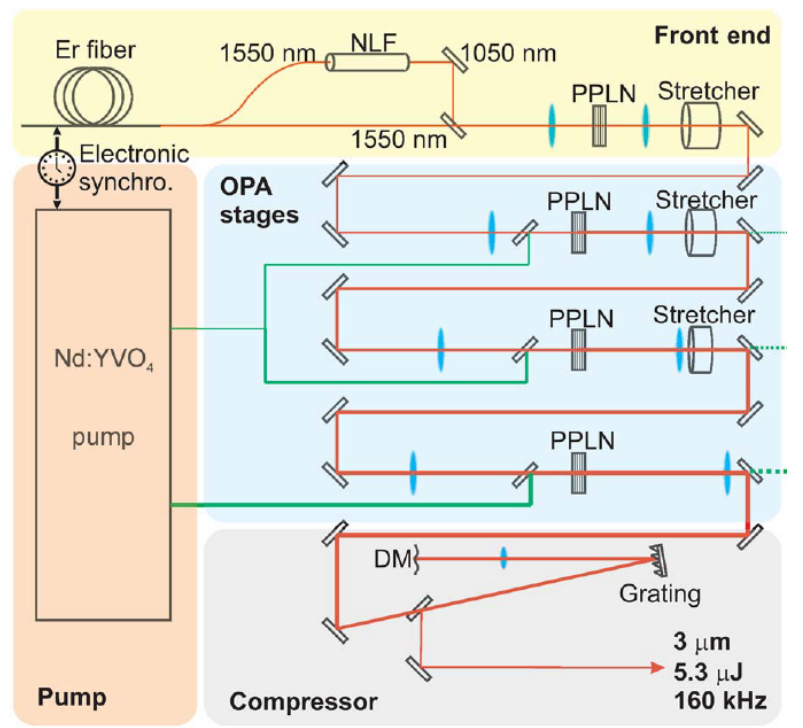

Figure 8. Scheme of the OPCPA system. NLF, Non-Linear Fiber; DM, Deformable Mirror; PPLN, Periodically Poled Lithium Niobate crystal. Adapted with permission from [50], Copyright OSA Publishing, 2011.

New perspectives in parametric amplifiers' development have opened with the introduction of two different approaches, namely waveform synthesizers and Frequency-domain Optical Parametric Amplifiers (FOPAs).

A promising route for the generation of extremely short optical pulses consists of synthesizing multiple sources. Synthesis is obtained by coherently combining the output of a number of different sources operating at different wavelength, so that a broader spectrum is constructed. The coherent combination of different near-IR OPCPAs, as well as of different fiber-based systems has been recently demonstrated [51,52], leading to sub-single cycle waveforms with an energy in the order of some tens of $\mu \mathrm{J}$. Systems based on this technology are suitable for energy scaling and can provide a solution for overcoming fundamental limitations, like limited parametric amplification bandwidth.

However, improved performances both in terms of scalability and pulse duration lead, in these cases, to an increased complexity in the setup. Indeed, synthesizing a sub-single cycle waveform requires both an accurate shaping of the pulses temporal profile and a full control on the CEPs and on the relative timing among the pulses. Acusto-optic programmable filters and balanced cross-correlators are used for properly shaping and timing the pulses with attosecond relative time stability.

A completely different scheme for achieving parametric amplification of CEP-stable pulses is Frequency-domain Optical Parametric Amplification (FOPA) [53].

As opposed to the previously described approach based on synthesis of multiple sources, FOPA consists of dividing the spectrum of seed pulses from a single parametric source and in amplifying each spectral component independently from the others. The working principle of FOPA is depicted in Figure 9a. Spectral partitioning is obtained by spreading the pulse spectrum over a plane, the Fourier Plane (FP), by means of a 4 -f setup. Then, different spectral components occupy a different position on the FP and can be amplified independently by using an array of non-linear crystals. Each element of this array is optimized for amplifying the corresponding spatial frequency, so that ultra-broadband phase matching is achieved, as shown in Figure 9b. As in the case of synthesizers, limitations in amplification bandwidth due to phase-matching in a single crystal are overcome. Moreover, since the incident energy is distributed over a large plane, seed and pump pulses with higher energy can be used, overcoming the restrictions imposed by limited crystal apertures. Up to now, $\sim 1.5-\mathrm{mJ}$ pulses at $1.8 \mu \mathrm{m}$ with a sub-two cycle duration have been produced at a $100-\mathrm{Hz}$ repetition rate by using the FOPA design [53]. 
(a)
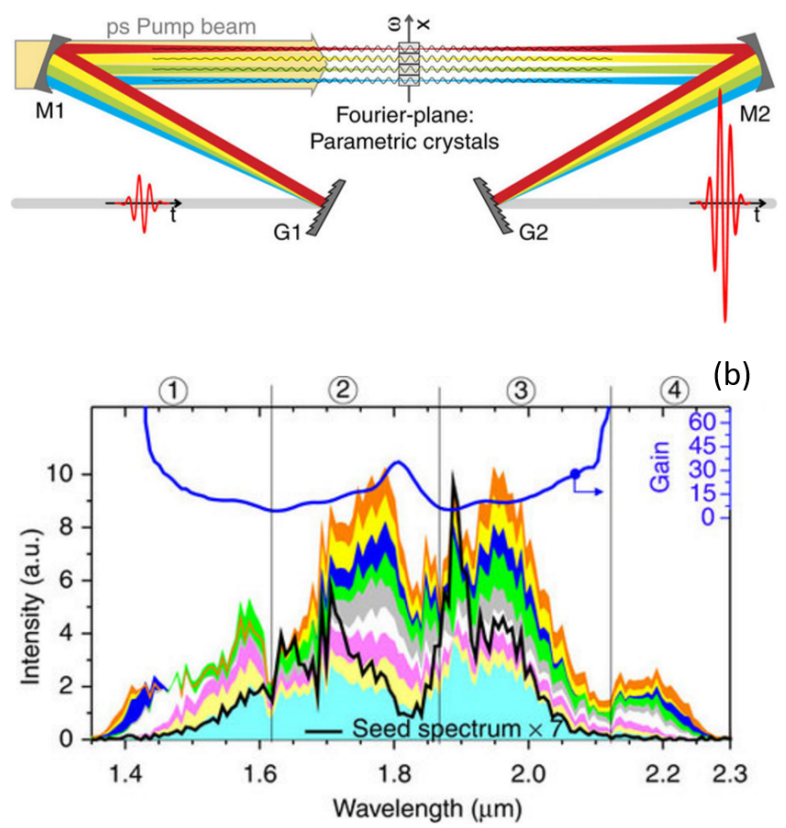

Figure 9. (a) Scheme of the Frequency-domain Optical Parametric Amplification (FOPA) system; (b) example of the amplified spectrum in the FOPA [53]. The black curve corresponds to the seed spectrum. The spectral ranges of the four BBO crystals used for the amplification in the Fourier plane are marked by vertical lines. The colored spectra correspond to amplification for different pump levels. The blue curve corresponds to the experimental gain curve. Adapted with permission from [53], Copyright NPG, 2014.

The development of powerful few-cycle parametric sources remains a challenging task from a technological point of view. Nevertheless, OPA and OPCPA systems will benefit from the introduction of the above-mentioned novel approaches towards the scalability of pulse energy and duration. Therefore, improvements are expected in the future both in terms of performances and in terms of system architecture.

\section{Applications}

The interest in developing ultrafast powerful laser sources lies in the possibility they offer to explore highly non-linear regimes of light-matter interaction, namely the strong-field regime and temporally-resolved phase transitions.

Strong-field phenomena are activated in matter undergoing interaction with an external electric field on the same strength-scale of the internal binding fields. This kind of phenomena thus involves a change in the structural properties of matter, mainly induced by the activation of electron excitation processes. The strong-field light-matter interaction regime is suitable for studying electron dynamics, as well as the corresponding structural evolution in atoms, molecules and solid-state systems. Up to now, the main experimental techniques used for accessing strong-field phenomena are: High-order Harmonic Generation (HHG) spectroscopy, photoelectron spectroscopy and pump-probe spectroscopy based on attosecond pulses.

Electron and nuclear dynamics occur on an ultrafast time-scale, ranging from hundreds of femtoseconds down to few attoseconds. Strong-field processes are thus deeply affected by the temporal properties of the exciting pulses, namely duration, field frequency (wavelength) and CEP.

In this sense, the use of CEP-stable few-cycle IR pulses leads to a number of significant advantages [54]. In particular, an electron interacting with an external field experiences a ponderomotive energy gain. This energy gain scales towards higher values when longer optical wavelengths (in the near-IR and 
mid-IR) are used. Higher electron energy corresponds to shorter De Broglie wavelength, which is useful for imaging small targets and for exciting transitions from deeply bound states.

Moreover, CEP-locked few-cycle pulses provide reproducible waveforms and can potentially be used to perform a fine control of ultrafast processes with a sub-cycle precision. High energy tunable few-cycle parametric sources in the IR spectral region allow one to fully exploit these advantages.

In this section, the main achievements in strong-field physics obtained by exploiting high-energy few-cycle near-IR and mid-IR sources will be discussed. In particular, attention is paid to reviewing very recent advances in HHG spectroscopy, photoelectron spectroscopy and attosecond science.

\subsection{High-Order Harmonic Generation Spectroscopy}

The HHG process is a highly non-linear light-matter interaction involving upconversion of the driving photon energy into higher values. In particular, emission of high-order harmonics of the fundamental one is observed. The discretization of the spectrum into harmonics derives from an interference process involving photon emission events occurring at different temporal windows. Indeed, light bursts of high energy photons are emitted by the target exposed to the radiation every half optical cycle of the laser field. The newly-generated spectral components provides coherent radiation in the extreme ultraviolet (XUV) and in the X-ray spectral region.

An easy-to-understand explanation of the harmonics emission process is provided by the so-called three-step model $[55,56]$. The three-step model is a semi-classical model that depicts HHG as a sequence of three steps: as a first step, tunnel ionization takes place, resulting in the ejection of an electron. The ejected electron is then accelerated by the external laser field, gaining kinetic energy. Because of the oscillating nature of the laser electric field, the accelerated electron has a non-negligible probability of recombining with the parent ion. Recombination is associated with a photon emission.

According to this picture, the maximum energy $\left(\hbar \omega_{m}\right)$ of the emitted photons is provided by the following expression: $\hbar \omega_{m}=I_{p}+3.17 U_{p}$, where $I_{p}$ is the ionization potential of the target and $U_{p}$ is the ponderomotive energy gained by the electron in the external field. The ponderomotive energy depends on the wavelength of the driving radiation, as $\lambda^{2}$. The increase in the electron energy comes at the cost of a dramatic reduction of the HHG efficiency due to a reduced recombination probability associated with the spreading of the electron wave-function. The HHG yield scales as $\approx \lambda^{-6}[57,58]$. As a result, in HHG experiments driven by IR pulses, the acquisition process takes longer since integration over a larger number of shots is required for achieving a suitable signal to noise ratio. In order to reduce measurement times in the long-wavelength regime, high repetition rate sources $(>1 \mathrm{kHz})$ have to be used.

Another factor affecting the HHG spectrum extension is phase matching. HHG takes place in an extended medium made up of a collection of dipoles, all acting as a source of XUV radiation. The spectrum of radiation emitted at a macroscopic level corresponds to that of a single dipole only if all emitters are in phase. Macroscopic effects in HHG are related to several factors: the focusing geometry of the driving pulses; the dispersion due to the plasma and neutral target; the nature of the sample (dipole contribution). Due to dispersion, phase-matching strongly depends on the wavelength of the driving radiation. When an unfavorable dispersion regime for the driving wavelength is attained, a suppression of the harmonics intensity and a reduction of the spectrum extension is observed [59].

HHG driven by near-IR and mid-IR pulses has been extensively studied in gas targets made up of atoms, molecules and clusters. In this spectral region, HHG does not suffer from strong macroscopic effects. Therefore, the advantage of generating an extended harmonics spectrum can be fully exploited, without incurring strong phase-matching limitations [60-62]. Besides spectral extension, few-cycle IR pulses are particularly useful for studying atoms, molecules and solids with transitional energies on the order of a few eV. In the HHG process, the excitation induced in the target involves a field-driven transition between bound and unbound states. When an exciting radiation with a photon energy much lower than the energy of the bound-unbound transition is used, direct ionization is less likely to occur. Direct ionization is detrimental for rescattering-based experiments since it is responsible for 
a reduction of the efficiency by depleting the ground state population and lowering the amount of possibly recolliding electrons.

In Figure 10a, the maximum ponderomotive energy acquired by electrons undergoing interaction with an external field is shown as a function of the radiation wavelength (for a fixed beam intensity). In this sense, a remarkable result was that achieved in 2012 by Popmintchev et al. [63]. Bright high-harmonic X-ray radiation with a spectrum extending up to $1.6 \mathrm{keV}(\lambda=7.7 \AA)$ was demonstrated by focusing $10-\mathrm{mJ}$ mid-IR pulses at $3.9 \mu \mathrm{m}$ into a hollow core fiber filled with He gas (see Figure 10b).
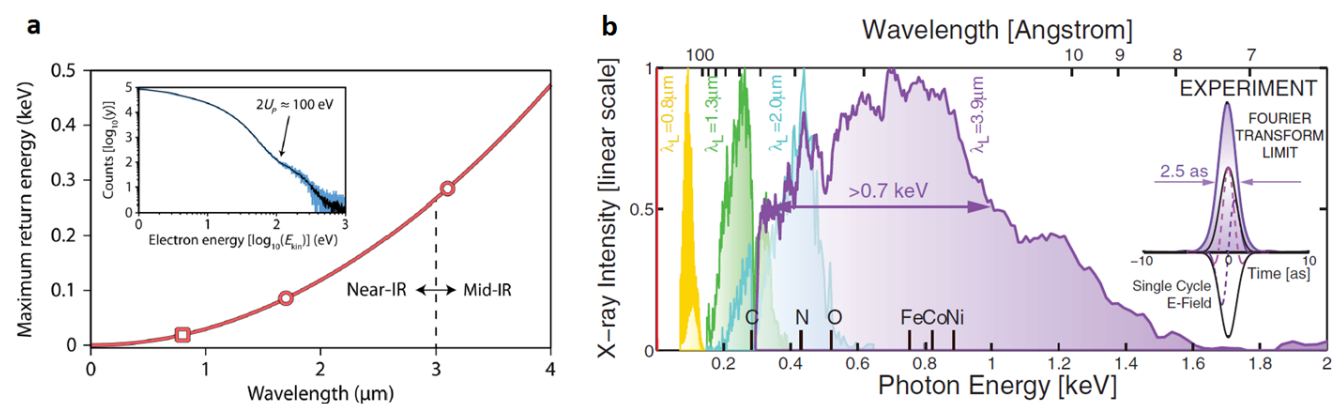

Figure 10. (a) Maximum electron return energy as a function of the wavelength for an intensity of $1.0 \times 10^{14} \mathrm{~W} / \mathrm{cm}^{2}$. In the inset, an electron energy distribution is shown, as measured after ionization of the $\mathrm{C}_{2} \mathrm{H}_{2}$ molecule using the $3.1 \mu \mathrm{m}$ radiation. Adapted with permission from [54], Copyright APS, 2015; (b) High-order harmonics spectral yield in xenon at different driving laser wavelengths $(0.8 \mu \mathrm{m}$, $1.3 \mu \mathrm{m}, 2 \mu \mathrm{m}$ and $3.9 \mu \mathrm{m})$. In the inset, the Fourier transform-limited pulse duration for the High-order Harmonic Generation (HHG) radiation driven by the 3.9- $\mu \mathrm{m}$ pulses is shown, corresponding to 2.5 attoseconds. Adapted with permission from [63], Copyright AAAS, 2012.

Due to the broad spectrum produced, extending up to the XUV and X-ray, the IR-driven HHG process can be used as a source for spectroscopic applications with elemental sensitivity [64]. We mention here the very recent achievement by Johnson and coworkers [65]. In their experiment, the high-order harmonics spectrum generated with a few-cycle 1.8- $\mu \mathrm{m}$ driving field was focused into a polythiophene sample, and the X-ray absorption near edge structure (XANES) response of the sample was acquired. X-ray spectroscopy is nowadays mainly performed at synchrotron and free-electron laser facilities, where an extremely bright radiation in the picosecond and femtosecond time-scale is produced. In this sense, the HHG-based setup currently provides an unsurpassed tabletop alternative to these large-scale infrastructures.

Besides providing a source of radiation for spectroscopy, HHG is in turn a spectroscopy technique itself, as the harmonics spectrum contains information about the structure and the dynamics occurring in the emitter.

As mentioned above, the use of IR pulses enables the increase of the electron energy up to the keV level. An electron energy of a few hundreds eV corresponds to a De Broglie wavelength of the order of a few $\AA$, on the same scale of the internuclear distances between atoms in molecules and solids. As a result, the spectrum of high-order harmonics is strongly affected by the structural properties of the emitter. Under appropriate inversion algorithms, an HHG-based tomographic reconstruction of the emitter can be performed with a sub-nanometer resolution [66]. Indeed, the structure of the Highest Occupied Molecular Orbital (HOMO) has been successfully probed in simple molecules by driving HHG with ultrashort IR light pulses [67-69].

As pointed out above, different components of the HHG spectrum are emitted at different times within a temporal window of the order of half optical cycle of the carrier frequency. A time-to-energy mapping is thus possible, and dynamics occurring on a sub-cycle time scale can potentially be recorded in the harmonics spectrum. 
Moreover, fingerprints of ultrafast electronic processes have been observed in HHG measurements. In particular, collective electron dynamics involving inner shell electrons have been observed in atoms by recording IR-driven high-order harmonics spectra extending beyond $100 \mathrm{eV}$. An enhancement in the high energy part of the spectrum (between $70 \mathrm{eV}$ and $100 \mathrm{eV}$ ) was recently observed in xenon by using few-cycle pulses at $1.8 \mu \mathrm{m}$ and $1.5 \mu \mathrm{m}[70,71]$. This spectral feature is attributed to a multi-electron effect known as giant resonance [72].

The role of ionization from multiple molecular orbitals has been studied in $\mathrm{CO}_{2}$ and $\mathrm{N}_{2}$ by performing HHG measurements at different pulse intensities and IR wavelengths [73,74]. Here, intensity-dependent spectral features are attributed to the increasing contribution of deeper orbitals, and wavelength-dependent spectral features are recognized as due to the interference among different ionization-recombination channels involving multiple orbitals. A comprehensive overview on the IR-driven HHG spectroscopy of atoms and molecules is provided in [11].

Efforts are currently devoted to the extension of HHG to the study of condensed-matter systems. Up to date achievements are briefly described below, and the main focus is placed on long-wavelengthdriven HHG experiments in bulk crystals. HHG in condensed matter systems is basically different from HHG in gas-phase samples. This is mainly because atoms are highly packed, and in the case of crystals, they are arranged to form a periodic lattice. Charges in crystals are delocalized over the entire lattice structure. As opposed to the atomic/molecular case, the HHG process cannot be described in terms of single electron trajectories, but in terms of charge oscillations. These collective charge motions are called dynamical Bloch oscillations.

The electronic properties of bulk materials derive from their band structure. In particular, the valence and the conduction bands are mainly responsible for the behavior of solids interacting with visible and IR radiation. In solid-state systems of technological interest, like semiconductors and insulators, the energy gap ranges from $0.1 \mathrm{eV}$ to $10 \mathrm{eV}$, thus covering from the mid-IR up to the EUV spectrum. The high harmonics emission in bulk material involves electrons and holes located respectively in the conduction and in the valence bands. In particular, this electron-hole population undergoes intraband acceleration by interaction with the external radiation. The resulting charge currents depends on the energy dispersion profile of the conduction and valence bands. Thus, the emitted HHG spectrum encodes information on the band structure from which it arises.

The generation of a high-order harmonics spectrum from solids is challenging because of a number of fundamental limitations. A first limitation is related to material ablation. When a driving radiation with an intensity above the material damage threshold is used, ablation takes place. Ablation leads to changes in the structural properties of the sample, thus preventing the observation of the effects correlated to the periodic arrangement of atoms. Since ablation is mainly induced by multi-photon absorption, the use of the IR long-wavelength is beneficial. Indeed, the lower is the driving photon energy, the lower is the probability of interband transition. A second limitation consists of the strong absorption of photons with energy higher than the band-gap. Due to the high density of the media, absorption of EUV photons becomes more critical than in gas samples, and it is responsible for a significant suppression of the harmonics signal. Sources with a high repetition rate are thus required for HHG experiments in condensed matter systems.

The first observation of a broad HHG spectrum from a solid target, extending well beyond the band-gap energy, was reported in 2010 by Ghimire et al. [75]. A few-cycle mid-IR parametric source was used to drive harmonics generation in a $\mathrm{ZnO}$ crystal (bandgap $\sim 3.2 \mathrm{eV}$ ). The source provides nine-cycle $\sim 100-\mu \mathrm{J}$ pulses, tunable from $3.2 \mu \mathrm{m}$ to $3.7 \mu \mathrm{m}$ (from $0.34 \mathrm{eV}$ to $0.38 \mathrm{eV}$ ), at a repetition rate of $1 \mathrm{kHz}$. High-order harmonics were observed extending up to $9.5 \mathrm{eV}$, which is more than $6 \mathrm{eV}$ above the bandgap. The interpretation of HHG spectra generated in bulk materials can be linked to the standard techniques available for HHG in gases, opening the way to the reconstruction of the band structure based on HHG spectroscopy [76].

An additional limiting issue in solid state HHG is ultrafast carrier scattering processes. Scattering leads to a disruption of the coherent charge transport under the external driving field, thus reducing 
HHG efficiency. In this sense, the possibility to control charges oscillations on an extremely ultrashort temporal window is promising, so as to preserve coherence. Sub-cycle confinement of intraband currents was achieved in $120 \mathrm{~nm}$-thin polycrystalline $\mathrm{SiO}_{2}$ films exposed to an intense ultrafast optical waveform generated in a visible light synthesizer [77]. Here, few-cycle to sub-cycle CEP-locked waveforms with a carrier frequency tunable in the visible spectral range were used.

The possibility to apply a similar approach to the study of materials of interest for technological applications is extremely attractive, such as low band-gap $(\sim 1 \mathrm{eV})$ semiconductors. In this context, pulses with a longer wavelength are required. Few-cycle CEP-stable sources in the IR to THz domain can provide a powerful tool for exploring these systems. It is worth mentioning here the results recently obtained by Schubert and coworkers [78]. In their experiment, they were capable of detecting HHG emission from bulk GaSe by using $30-\mathrm{THz}$ waveforms with a hundred-femtosecond duration. In particular, harmonics in the terahertz-to-visible spectral region were detected.

HHG-based experiments in condensed matter systems are of crucial interest for technological applications, paving the way for the development of XUV photonics as a tool for controlling and manipulating charge transport dynamics on an attosecond time scale.

\subsection{Attosecond Pulse Generation}

The HHG process is at the base of attosecond pulse generation. As discussed in the previous section, this process allows for the emission of a broad spectrum of radiation as a result of an upconversion mechanism for which high-order harmonics of the driving pulse frequency are generated. Such an extended spectrum supports pulse duration on the attosecond time scale.

As we have already discussed, the HHG spectrum depends on the wavelength of the driving radiation, so that the longer is the wavelength, the broader is the spectrum. In particular, when near-IR and mid-IR pulses are used, a harmonics signal can be obtained, extending up to the soft X-ray spectral region. Thus, long-wavelength driving pulses provide the advantage of supporting the generation of attosecond pulses shorter than those achievable using common Ti:Sa 800-nm driving pulses. Moreover, the soft X-ray spectral window is attained, which is particularly attractive for spectroscopy. X-ray-based attosecond pulses can be exploited for studying electron dynamics involving deep electronic states in atomic, molecular and condensed-matter systems.

An additional benefit, connected to the use of pulses in the long-wavelength range, is related to the temporal structure of the high-order harmonics radiation that is affected by a non-linear temporal dispersion, known as attochirp. Attochirp arises from the temporal distribution of the ponderomotive energies acquired by the electrons under the effect of the driving field. This chirp depends on the wavelength of the driving radiation according to an inverse scaling law. As a consequence, long-wavelength pulses allow for a reduction of the attochirp, thus helping in the generation of transform-limited attosecond pulses [79].

In HHG, the emission of XUV and X-ray radiation is distributed all along the driving pulse duration. However, in order to obtain a single attosecond pulse for each driving pulse (namely an Isolated Attosecond Pulse (IAP)), emission from a single half-cycle of the driving field must be selected. Selection can be achieved either by confining radiation in a sub-cycle temporal window or by filtering a well-defined portion of the HHG spectrum.

In the first case, a control on the HHG process is required, which can be achieved by properly tailoring the driving pulse waveform. For this purpose, a number of techniques have been developed, exploiting the dependence of HHG on pulse polarization [80,81] and field amplitude profile [82]. In the second case, a high pass filter is applied to the harmonics spectrum [83]. Indeed, if few-cycle driving pulses are used, the high-energy part of the harmonics emission can be attributed to a single half-cycle, the one in which the amplitude gets its maximum value.

All of these techniques are implemented by means of few-cycle CEP-stable driving pulses. Indeed, few cycle pulses provide a higher peak-to-peak contrast among successive optical cycles, thus favoring 
single half-cycle selection. Moreover, for optimizing such a contrast, the CEP has to be locked to a proper value.

So far, IAPs at $800 \mathrm{~nm}$ have been successfully generated by means of the above-mentioned schemes. The extension to near-IR and mid-IR window is still under investigation, the main limiting factor being the low HHG efficiency at a longer wavelength. In this framework, the development of powerful parametric sources operating in these spectral regions has recently driven research. In the last few years, a number of results have been obtained demonstrating IAPs' emission in the long-wavelength range. The first experimental characterization of isolated attosecond pulses driven by $1.7 \mu \mathrm{m}$ was recently published by Saito and coworkers [84].

Before Saito's works, fingerprints of IAPs have been already observed in HHG experiments performed with IR pulses. Millijoule-level few-cycle CEP-stable pulses at $1.5 \mu \mathrm{m}$ were used in combination with 800-nm pulses for performing HHG in noble gas [85,86]. In these experiments, a parallel and a perpendicular polarization configuration was explored for the two colors. In both cases, an XUV supercontinuum spectrum, extending above $100 \mathrm{eV}$, was measured. The lack of harmonics features in the HHG spectrum can be unequivocally attributed to a single burst of XUV emission with a potential attosecond duration. A similar approach, based on the superposition of two few-cycle CEP-stable mid-IR laser pulses with different wavelengths $(1.35 \mu \mathrm{m}, 1.75 \mu \mathrm{m})$, was successfully implemented $[87,88]$.

In 2012, a new scheme for IAPs generation was proposed, named attosecond lighthouse [89]. Experimental demonstrations were soon after performed $[90,91]$ by using $800-\mathrm{nm}$ laser pulses. The attosecond lighthouse method is based on the selection of an IAP within the train of XUV bursts by imprinting each of them with a different propagation direction. This can be obtained by acting on the space-time properties of the driving pulse, specifically by rotating the wavefront. As a result, a different front angle can be associated with each half-cycle of the pulse. Attosecond bursts are thus emitted at different angles, and by spatially selecting a well-defined direction of propagation, IAPs can be obtained. Experimental evidence of IR-driven IAPs' generation were reported in 2015 by Silva et al. [92] and Zhang et al. [93]. In both cases, 13-fs pulses at $\sim 1.8 \mu \mathrm{m}$ with an energy on the hundreds- $\mu \mathrm{J}$ level were uses. The availability of a $\mathrm{kHz}$ repetition rate parametric system allowed Silva and coworkers to detect an extremely broad XUV emission, up to the carbon K-edge (284 eV), potentially supporting 30 -as pulse duration.

As already said, a remarkable progress in isolated attosecond pulse generation in the long wavelength regime is provided by the work in [84]. Here, a complete temporal characterization of IAPs generated by means of few-cycle CEP-stable parametric pulses in the near-IR was reported for the first time. A $1-\mathrm{kHz}$ OPCPA system delivering a 12.7-fs pulse at $1.7 \mu \mathrm{m}$ with an energy of $1.5 \mathrm{~mJ}$ was used. A fraction of the pulse energy, $570 \mu \mathrm{m}$, was focused into a gas cell filled with Ar, and XUV emission was detected up to $100 \mathrm{eV}$. The high-energy part of the spectrum was selected $(90 \mathrm{eV})$ by means of a Mo/Si mirror. Spectral selection led to the isolation of a single burst of XUV radiation.

The attosecond streaking technique was then used for retrieving the amplitude and the phase of the IAP. For this characterization, the XUV pulse in combination with the co-propagating $1.7-\mu \mathrm{m}$ fundamental pulse is sent into a neon gas jet. The XUV pulse ionizes the target gas, generating a photoelectron wave packet, which is a replica of the XUV pulse. The photoelectron energy is modulated by the interaction with the IR streaking pulse. The photoelectron energy distribution is acquired by means of a time-of-flight spectrometer as a function of the delay between the two pulses. In such a away, a streaking map is obtained (Figure 11a) encoding information on the attosecond pulse temporal profile, as shown in Figure 11b. This experiment provided the first demonstration of photoelectron streaking measurement in the near-IR domain, opening the way towards ultrafast soft X-ray photoelectron spectroscopy. 

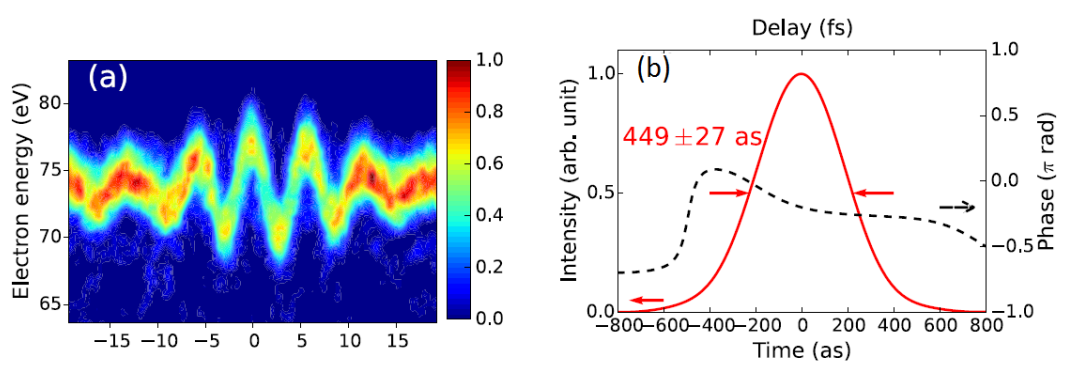

Figure 11. (a) Measured streaking spectrograms; (b) retrieved temporal profile of the XUV pulse. Adapted with permission from [84], Copyright NPG, 2016.

\subsection{Photoelectron Spectroscopy}

As described in the HHG section, strong fields can induce tunnel ionization in matter, leading to the emission of photoelectrons. The energy and momentum distributions of photoelectrons encode structural and dynamical information on the target. Photoelectrons' detection is thus at the base of a number of spectroscopy techniques. For instance, photoelectrons are widely used for probing structural properties of matter at the atomic scale, in diffraction-based imaging methods. The main advantage of using electrons as a probe lies in their high cross-section of interaction with atoms. Indeed, electrons are charged particles, and their interaction with nuclei and electronic distributions is significantly enhanced with respect to photons. As an example, for energy values in the X-ray spectral window, the photoelectron scattering cross-section is more than five orders of magnitude higher than that of photons [94]. In order to attain sub-nanometer resolution, photoelectrons with an energy of the order of a few hundred $\mathrm{eV}$ are required. Ponderomotive acceleration of photoelectrons under high energy IR laser pulses provides the possibility to access such an extreme energy regime. Moreover, by using a few cycle pulses, ultrafast dynamics occurring into the sample can be probed with a sub-cycle resolution.

In a strong field experiment, photo-emitted electrons are steered by the external driving field. Depending on the initial conditions (namely, instant of emission and initial momentum), photoelectrons can follow a number of different field-induced trajectories. As a first scenario, they may be driven away from the system they were emitted from, contributing to a process named Above Threshold Ionization (ATI). As an opposite scenario, they may be driven back to the parent ion. In this case, different processes may happen upon return. Recollision with the ion can be responsible for photo-recombination, resulting in the emission of high-order harmonic radiation. Photoelectrons may otherwise experience elastic or inelastic scattering, associated with momentum exchange and ion excitation.

Photoelectrons undergoing different processes provide a different informational content. The main achievements obtained by studying photoelectrons in an IR-driven strong-field regime are reported below.

In the last few years, remarkable results in molecular imaging have been obtained by applying a photoelectron-based diffraction imaging technique, named Laser-Induced Electron Diffraction (LIED) [95]. LIED allows to extract structural features of gas-phase molecules directly from electrons, which are elastically scattered off the target. In particular, high energy electrons $(>100 \mathrm{eV})$ steered along the laser polarization direction are selected, and their momentum distribution is detected. The structural properties of molecular scatterers can be retrieved by properly processing this momentum map.

In 2012, Blaga and coworkers exploited high energy few-cycle pulses tunable in the IR for implementing time-resolved LIED in simple molecules. They succeeded in capturing the dynamical evolution of oxygen and nitrogen structures under a vibrational excitation [96]. Recently, advances have been reported in LIED experiments performed with mid-infrared few-cycle high repetition rate 
parametric sources, providing the extension of this technique to the sub- $\AA$ imaging of a polyatomic molecule, such as ethylene $[97,98]$, and to the exploration with a sub-fs resolution of a chemical process, such as bond-breaking in acetylene [99].

In diffraction experiments, the information on the phase of the diffracted beam is lost. A complete amplitude-phase characterization can in principle be obtained by using a strong-field technique inspired by classical holography, namely Strong-Field Photoelectron Holography (SFPH) $[100,101]$. The SFPH technique differs from LIED since it is based on the acquisition of an interference pattern, which is due to the superposition of a scattered and an unscattered photoelectron wave packet, both emitted from the same site by laser-induced tunnel ionization. Proof of principle of photoelectron holography was provided in 2011 by Y . Huismans et al. [102] with the study of metastable xenon atoms with a hundred-fs Free Electron Laser (FEL) beam at $7 \mu \mathrm{m}$. Subsequently, a Ti:Sa femtosecond laser system, delivering 40 -fs pulses at $800 \mathrm{~nm}$, was used for implementing SFPH in $N_{2}$ [103]. Up to now, the possibility to extend SFPH to the few-cycle mid-IR regime is still under investigation [104]. Moreover, the few-cycle regime would allow one to confine photoelectrons, steering motion into a short temporal window, thus preventing multiple rescattering events and simplifying phase-retrieval procedures. Thus, few-cycle mid-IR parametric sources can currently be addressed as the most promising table-top alternative to FELs in SFPH experiments.

Strong-field processes are intrinsically connected to the control of electron currents at an atomic scale with sub-optical cycle temporal resolution. The perspective for future technological applications in photonic devices is promoting research on strong-field phenomena at the condensed-matter level. The possibility to access this regime of interaction is however limited by the necessity to use an exciting radiation with an intensity below the material damage threshold. As mentioned in the HHG section, the use of mid-IR is beneficial, hindering multiphoton absorption and, thus, optical damage. Moreover, plasmonic nanostructures have been identified as ideal candidate for supporting strong-field physics in condensed-matter systems, as field confinement can lead to a strong field regime even with a lower intensity driving radiation.

Recently, experimental results have been published demonstrating strong-field photoemission from plasmonic nanostructures in a non-destructive regime by means of few-cycle mid-IR pulses.

Herink and coworkers [105] observed sub-cycle photoelectron emission from isolated metal nano-tips over a broad spectral window, extending up to hundreds of $\mathrm{eV}$. A femtosecond source tunable in the near-IR and in the mid-IR was used for exciting photoemission and for triggering space-charge effects in the nanostructures. The sub-wavelength field confinement at the tip was here identified as responsible for a huge enhancement of the kinetic energy of photoelectrons escaping from the metal. The experimental and simulated kinetic energy distributions of photoelectrons acquired at a number of IR field intensities for a driving wavelength of $3.8 \mu \mathrm{m}$ are shown in Figure 12a.

a

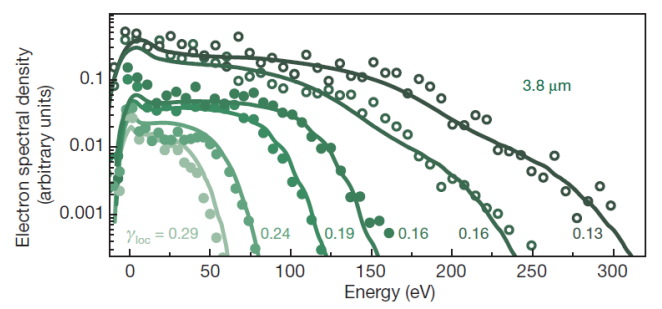

b

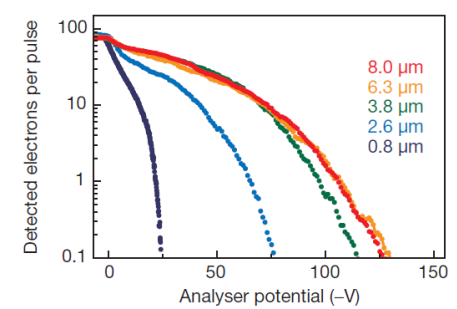

Figure 12. (a) Experimental (circles) and simulated (solid lines) kinetic energy distributions of photoelectrons emitted from the nano-tips for increasing intensities at a wavelength of $3.8 \mu \mathrm{m}$; (b) Electron energy distribution as a function of the wavelength. Curves represent the number of electrons with energies above the analyzer potential. Adapted with permission from [105], Copyright NPG, 2012. 
In addition, measurements at various wavelengths were performed in order to investigate the scaling dynamics of photoelectron kinetic energy (see Figure 12b). In this sense, an anomalous behavior was observed for increasing wavelength. Indeed, for wavelengths longer than $3.8 \mu \mathrm{m}$, a new sub-cycle regime was identified, in which the photoelectrons' spectrum becomes substantially wavelength independent. The transition to this wavelength independent regime was attributed to the local field distribution, decaying over distances shorter than the photoelectron quiver amplitude. Apart from plasmon-assisted strong-field experiments, this regime is proven to be widely inaccessible because of the diffraction limit affecting the beam dimension. In this framework, strong-field photoemission under an IR excitation can be addressed as a potential instruments for probing nano-localized field distributions and their ultrafast dynamical evolution.

Strong-field photoemission was subsequently observed by Teichmann et al. [106] also in plasmonic films under exposition to femtosecond, mid-infrared pulses provided by an OPCPA source at $3.1 \mu \mathrm{m}$. Tunneling emission was here demonstrated at the considerably low intensity of $\sim 1 \mathrm{GW} / \mathrm{cm}^{2}$, with the localized plasmon field being responsible for an extension of the photoelectron energy spectrum up to two orders of magnitude.

\subsection{Other Fields of Application}

As a remarkable example of additional fields of application for high-energy few-cycle IR parametric sources, we mention here the perspective of using IR-driven HHG radiation for seeding the Free Electron Laser (FEL) system [107]. In this sense, the benefits of using a tunable IR parametric source as a front-end lies in the possibility to generate an extremely extended seeding spectrum of FEL radiation, as well as in the possibility to finely tune FEL harmonics spectral position.

IR parametric sources providing ultrashort pulses are becoming a promising tool also in a number of sectors, which are not directly related to the ultrafast realm. In particular, the high energy flux provided by few-cycle pulses combined with the long penetration depth exhibited by the IR radiation in a number of materials of technological interest (semiconductors and insulators) can be exploited for manipulating matter at a microscopic level. Recently, Austin and coworkers observed High Spatial Frequency Laser-induced periodic surface structures (HSFL) on a germanium surface exposed to a femtosecond sub-bandgap intense radiation [108]. These spatial features result from a surface scattering mechanism; namely, the interference between the incident radiation and the scattered fraction leads to an intensity modulation along the surface, resulting in a periodic pattern of micro-stripes. This technique is potentially applicable to any surface and can be used in micro-fabrication processes.

Finally, the attractive perspective of applying femtosecond sources working in the long-wavelength domain for achieving intracellular micro- and nano-surgery is also under investigation [109].

\section{Conclusions}

This paper is intended as a review of the state of the art in the field of high energy ultrafast IR parametric amplifiers. The most advanced systems based on OPA and on OPCPA design have been described, by pointing out their key features. Promising perspectives for the development of novel architectures with improved performances have been discussed, based on new technical solutions, such as spectral synthesis and frequency domain amplification. Finally, an overview of the main fields of applications in strong field physics has been provided, including HHG spectroscopy, attosecond science and photoelectron spectroscopy.

Acknowledgments: The research leading to these results has received funding from the European Research Council Starting Research Grant UDYNI (Grant Agreement No. 307964), from the European Union Horizon 2020 research and innovation program under the Marie Sklodowska-Curie Grant Agreement No. 641789 MEDEA, from the Italian Ministry of Research and Education (ELI project-ESFRI Roadmap), from Regione Lombardia with the project FEMTOTERA (ID: CONCERT2014-008) and from Fondazione Cariplo with the project GREENS (No. 2013-0656). 
Author Contributions: A.G.C. wrote the manuscript, C.V. and S.S. coordinated the work, A.G.C., M.N., M.D. developed the OPA source described in the manuscript and performed the characterization measurements. All the authors reviewed and provided comments on the manuscript.

Conflicts of Interest: The authors declare no conflict of interest.

\section{References}

1. Keller, U. Recent developments in compact ultrafast lasers. Nature 2003, 424, 831-838.

2. Strickland, D.; Mourou, G. Compression of amplified chirped optical pulses. Opt. Commun. 1985, 56, 219-221.

3. Brabec, T.; Krausz, F. Intense few-cycle laser fields: Frontiers of nonlinear optics. Rev. Mod. Phys. 2000, $72,545$.

4. Manzoni, C.; Cerullo, G. Design criteria for ultrafast optical parametric amplifiers. J. Opt. 2016, $18,103501$.

5. Giordmaine, J.; Miller, R.C. Tunable Coherent Parametric Oscillation in $\mathrm{LiNbO}_{3}$ at Optical Frequencies. Phys. Rev. Lett. 1965, 14, 973.

6. Baumgartner, R.; Byer, R. Optical parametric amplification. IEEE J. Quant. Electron. 1979, 15, $432-444$.

7. Danielius, R.; Banfi, G.; Di Trapani, P.; Righini, R.; Piskarskas, A.; Stabinis, A. Traveling-wave parametric generation of widely tunable, highly coherent femtosecond light pulses. JOSA B 1993, 10, 2222-2232.

8. Cerullo, G.; De Silvestri, S. Ultrafast optical parametric amplifiers. Rev. Sci. Instrum. 2003, 74, 1-18.

9. Brida, D.; Manzoni, C.; Cirmi, G.; Marangoni, M.; Bonora, S.; Villoresi, P.; De Silvestri, S.; Cerullo, G. Few-optical-cycle pulses tunable from the visible to the mid-infrared by optical parametric amplifiers. J. Opt. 2009, 12, 013001.

10. Tajima, T.; Mourou, G. Zettawatt-exawatt lasers and their applications in ultrastrong-field physics. Phys. Rev. Spec. Top. Accel. Beams 2002, 5, 031301.

11. Vozzi, C.; Negro, M.; Stagira, S. Strong-field phenomena driven by mid-infrared ultrafast sources: JMO Series: Attosecond and Strong Field Science. J. Mod. Opt. 2012, 59, 1283-1302.

12. Backus, S.; Durfee, C.G., III; Murnane, M.M.; Kapteyn, H.C. High power ultrafast lasers. Rev. Sci. Instrum. 1998, 69, 1207-1223.

13. Dubietis, A.; Butkus, R.; Piskarskas, A.P. Trends in chirped pulse optical parametric amplification. IEEE J. Sel. Top. Quant. Electron. 2006, 12, 163.

14. Harris, S.; Oshman, M.; Byer, R. Observation of tunable optical parametric fluorescence. Phys. Rev. Lett. 1967, 18, 732 .

15. Banfi, G.; Danielius, R.; Di Trapani, P.; Foggi, P.; Righini, R.; Piskarskas, A. Femtosecond traveling-wave parametric generation with lithium triborate. Opt. Lett. 1993, 18, 1633-1635.

16. Manzoni, C.; Cirmi, G.; Brida, D.; De Silvestri, S.; Cerullo, G. Optical-parametric-generation process driven by femtosecond pulses: Timing and carrier-envelope phase properties. Phys. Rev. A 2009, 79, 033818.

17. Alfano, R.; Shapiro, S. Observation of self-phase modulation and small-scale filaments in crystals and glasses. Phys. Rev. Lett. 1970, 24, 592.

18. Bellini, M.; Hänsch, T.W. Phase-locked white-light continuum pulses: Toward a universal optical frequency-comb synthesizer. Opt. Lett. 2000, 25, 1049-1051.

19. Baum, P.; Lochbrunner, S.; Piel, J.; Riedle, E. Phase-coherent generation of tunable visible femtosecond pulses. Opt. Lett. 2003, 28, 185-187.

20. Baum, P.; Riedle, E.; Greve, M.; Telle, H.R. Phase-locked ultrashort pulse trains at separate and independently tunable wavelengths. Opt. Lett. 2005, 30, 2028-2030.

21. Baltuška, A.; Fuji, T.; Kobayashi, T. Controlling the carrier-envelope phase of ultrashort light pulses with optical parametric amplifiers. Phys. Rev. Lett. 2002, 88, 133901.

22. Fuji, T.; Rauschenberger, J.; Apolonski, A.; Yakovlev, V.S.; Tempea, G.; Udem, T.; Gohle, C.; Hänsch, T.W.; Lehnert, W.; Scherer, M.; et al. Monolithic carrier-envelope phase-stabilization scheme. Opt. Lett. 2005, 30, 332-334.

23. Vozzi, C.; Cirmi, G.; Manzoni, C.; Benedetti, E.; Calegari, F.; Sansone, G.; Stagira, S.; Svelto, O.; Silvestri, S.D.; Nisoli, M.; et al. High-energy, few-optical-cycle pulses at $1.5 \mu \mathrm{m}$ with passive carrier-envelope phase stabilization. Opt. Express 2006, 14, 10109-10116.

24. Koechner, W. Solid-State Laser Engineering; Springer: New York, NY, USA, 2013; Volume 1. 
25. Pires, H.; Baudisch, M.; Sanchez, D.; Hemmer, M.; Biegert, J. Ultrashort pulse generation in the mid-IR. Progr. Quant. Electron. 2015, 43, 1-30.

26. Mason, P.D.; Michaille, L.F. Review of the development of nonlinear materials for mid-IR generation. In Proceedings of the SPIE Europe Security and Defence, Cardiff, UK, 15 September 2008; p. 71150N.

27. Jiang, X.M.; Guo, S.P.; Zeng, H.Y.; Zhang, M.J.; Guo, G.C. Large crystal growth and new crystal exploration of mid-infrared second-order nonlinear optical materials. In Structure-Property Relationships in Non-Linear Optical Crystals II; Springer: New York, NY, USA, 2012; pp. 1-43.

28. Vozzi, C.; Calegari, F.; Benedetti, E.; Gasilov, S.; Sansone, G.; Cerullo, G.; Nisoli, M.; Silvestri, S.D.; Stagira, S. Millijoule-level phase-stabilized few-optical-cycle infrared parametric source. Opt. Lett. 2007, 32, 2957-2959.

29. Vozzi, C.; Manzoni, C.; Calegari, F.; Benedetti, E.; Sansone, G.; Cerullo, G.; Nisoli, M.; Silvestri, S.D.; Stagira, S. Characterization of a high-energy self-phase-stabilized near-infrared parametric source. J. Opt. Soc. Am. B 2008, 25, B112-B117.

30. Hauri, C.; Kornelis, W.; Helbing, F.; Heinrich, A.; Couairon, A.; Mysyrowicz, A.; Biegert, J.; Keller, U. Generation of intense, carrier-envelope phase-locked few-cycle laser pulses through filamentation. Appl. Phys. B 2004, 79, 673-677.

31. Shirakawa, A.; Sakane, I.; Kobayashi, T. Pulse-front-matched optical parametric amplification for sub-10-fs pulse generation tunable in the visible and near infrared. Opt. Lett. 1998, 23, 1292-1294.

32. Hauri, C.P.; Lopez-Martens, R.B.; Blaga, C.I.; Schultz, K.D.; Cryan, J.; Chirla, R.; Colosimo, P.; Doumy, G.; March, A.M.; Roedig, C.; et al. Intense self-compressed, self-phase-stabilized few-cycle pulses at $2 \mu \mathrm{m}$ from an optical filament. Opt. Lett. 2007, 32, 868-870.

33. Schmidt, B.E.; Shiner, A.D.; Lassonde, P.; Kieffer, J.C.; Corkum, P.B.; Villeneuve, D.M.; Légaré, F. CEP stable 1.6 cycle laser pulses at $1.8 \mu \mathrm{m}$. Opt. Express 2011, 19, 6858-6864.

34. Schmidt, B.E.; Béjot, P.; Giguère, M.; Shiner, A.D.; Trallero-Herrero, C.; Bisson, E.; Kasparian, J.; Wolf, J.P.; Villeneuve, D.M.; Kieffer, J.C.; et al. Compression of $1.8 \mu \mathrm{m}$ laser pulses to sub two optical cycles with bulk material. Appl. Phys. Lett. 2010, 96, 121109, doi:10.1063/1.3359458.

35. Wang, H.; Chini, M.; Moon, E.; Mashiko, H.; Li, C.; Chang, Z. Coupling between energy and phase in hollow-core fiber based f-to-2f interferometers. Opt. Express 2009, 17, 12082-12089.

36. Dubietis, A.; Jonušauskas, G.; Piskarskas, A. Powerful femtosecond pulse generation by chirped and stretched pulse parametric amplification in BBO crystal. Opt. Commun. 1992, 88, $437-440$.

37. Piskarskas, A.; Stabinis, A.; Yankauskas, A. Phase phenomena in parametric amplifiers and generators of ultrashort light pulses. Sov. Phys. Uspekhi 1986, 29, 869.

38. Mücke, O.D.; Ališauskas, S.; Verhoef, A.J.; Pugžlys, A.; Baltuška, A.; Smilgevičius, V.; Pocius, J.; Giniūnas, L.; Danielius, R.; Forget, N. Self-compression of millijoule $1.5 \mu \mathrm{m}$ pulses. Opt. Lett. 2009, 34, 2498-2500.

39. Mücke, O.D.; Sidorov, D.; Dombi, P.; Pugžlys, A.; Baltuška, A.; Ališauskas, S.; Smilgevičius, V.; Pocius, J.; Giniūnas, L.; Danielius, R.; et al. Scalable Yb-MOPA-driven carrier-envelope phase-stable few-cycle parametric amplifier at $1.5 \mu \mathrm{m}$. Opt. Lett. 2009, 34, 118-120.

40. Ishii, N.; Kaneshima, K.; Kanai, T.; Watanabe, S.; Itatani, J. Generation of ultrashort intense optical pulses at $1.6 \mu \mathrm{m}$ from a bismuth triborate-based optical parametric chirped pulse amplifier with carrier-envelope phase stabilization. J. Opt. 2015, 17, 094001.

41. Fuji, T.; Ishii, N.; Teisset, C.Y.; Gu, X.; Metzger, T.; Baltuska, A.; Forget, N.; Kaplan, D.; Galvanauskas, A.; Krausz, F. Parametric amplification of few-cycle carrier-envelope phase-stable pulses at $2.1 \mu \mathrm{m}$. Opt. Lett. 2006, 31, 1103-1105.

42. Gu, X.; Marcus, G.; Deng, Y.; Metzger, T.; Teisset, C.; Ishii, N.; Fuji, T.; Baltuska, A.; Butkus, R.; Pervak, V.; et al. Generation of carrier-envelope-phase-stable 2-cycle $740-\mu \mathrm{J}$ pulses at 2.1- $\mu \mathrm{m}$ carrier wavelength. Opt. Express 2009, 17, 62-69.

43. Chalus, O.; Bates, P.K.; Smolarski, M.; Biegert, J. Mid-IR short-pulse OPCPA with micro-Joule energy at $100 \mathrm{kHz}$. Opt. Express 2009, 17, 3587-3594.

44. Erny, C.; Heese, C.; Haag, M.; Gallmann, L.; Keller, U. High-repetition-rate optical parametric chirped-pulse amplifier producing 1- $\mu \mathrm{J}$, sub-100-fs pulses in the mid-infrared. Opt. Express 2009, 17, 1340-1345.

45. Andriukaitis, G.; Balčiūnas, T.; Ališauskas, S.; Pugžlys, A.; Baltuška, A.; Popmintchev, T.; Chen, M.C.; Murnane, M.M.; Kapteyn, H.C. 90 GW peak power few-cycle mid-infrared pulses from an optical parametric amplifier. Opt. Lett. 2011, 36, 2755-2757. 
46. Sorokina, I.T.; Dvoyrin, V.V.; Tolstik, N.; Sorokin, E. Mid-IR ultrashort pulsed fiber-based lasers. IEEE J. Sel. Top. Quant. Electron. 2014, 20, 99-110.

47. Rudd, J.; Law, R.; Luk, T.; Cameron, S. High-power optical parametric chirped-pulse amplifier system with a $1.55 \mu \mathrm{m}$ signal and a $1.064 \mu \mathrm{m}$ pump. Opt. Lett. 2005, 30, 1974-1976.

48. Kraemer, D.; Cowan, M.L.; Hua, R.; Franjic, K.; Miller, R.D. High-power femtosecond infrared laser source based on noncollinear optical parametric chirped pulse amplification. JOSA B 2007, 24, 813-818.

49. Chalus, O.; Thai, A.; Bates, P.; Biegert, J. Six-cycle mid-infrared source with $3.8 \mu \mathrm{J}$ at $100 \mathrm{kHz}$. Opt. Lett. 2010, $35,3204-3206$.

50. Thai, A.; Hemmer, M.; Bates, P.; Chalus, O.; Biegert, J. Sub-250-mrad, passively carrier-envelope-phase-stable mid-infrared OPCPA source at high repetition rate. Opt. Lett. 2011, 36, 3918-3920.

51. Huang, S.W.; Cirmi, G.; Moses, J.; Hong, K.H.; Bhardwaj, S.; Birge, J.R.; Chen, L.J.; Li, E.; Eggleton, B.J.; Cerullo, G.; et al. High-energy pulse synthesis with sub-cycle waveform control for strong-field physics. Nat. Photonics 2011, 5, 475-479.

52. Krauss, G.; Lohss, S.; Hanke, T.; Sell, A.; Eggert, S.; Huber, R.; Leitenstorfer, A. Synthesis of a single cycle of light with compact erbium-doped fibre technology. Nat. Photonics 2010, 4, 33-36.

53. Schmidt, B.E.; Thiré, N.; Boivin, M.; Laramée, A.; Poitras, F.; Lebrun, G.; Ozaki, T.; Ibrahim, H.; Légaré, F. Frequency domain optical parametric amplification. Nat. Commun. 2014, 5, doi:10.1038/ncomms4643.

54. Wolter, B.; Pullen, M.G.; Baudisch, M.; Sclafani, M.; Hemmer, M.; Senftleben, A.; Schröter, C.D.; Ullrich, J.; Moshammer, R.; Biegert, J. Strong-field physics with mid-IR fields. Phys. Rev. X 2015, 5, 021034.

55. Corkum, P.B. Plasma perspective on strong field multiphoton ionization. Phys. Rev. Lett. 1993, 71, $1994-1997$.

56. Schafer, K.J.; Krause, J.L.; Kulander, K.C. Short-Pulse High-Intensity Lasers and Applications. In Proceedings of the 4th Society of Photo-Optical Instrumentation Engineers (SPIE) international symposium, Los Angeles, CA, USA, 20-25 January 1991; SPIE Society of Photo-Optical Instrumentation Engineers: Bellingham, WA, USA; Volume 1860, pp. 190-197.

57. Tate, J.; Auguste, T.; Muller, H.G.; Salières, P.; Agostini, P.; DiMauro, L.F. Scaling of Wave-Packet Dynamics in an Intense Midinfrared Field. Phys. Rev. Lett. 2007, 98, 013901.

58. Shiner, A.D.; Trallero-Herrero, C.; Kajumba, N.; Bandulet, H.C.; Comtois, D.; Légaré, F.; Giguère, M.; Kieffer, J.C.; Corkum, P.B.; Villeneuve, D.M. Wavelength Scaling of High Harmonic Generation Efficiency. Phys. Rev. Lett. 2009, 103, 073902.

59. Balcou, P.; Salières, P.; L'Huillier, A.; Lewenstein, M. Generalized phase-matching conditions for high harmonics: The role of field-gradient forces. Phys. Rev. A 1997, 55, 3204-3210.

60. Yakovlev, V.S.; Ivanov, M.; Krausz, F. Enhanced phase-matching for generation of soft X-ray harmonics and attosecond pulses in atomic gases. Opt. Express 2007, 15, 15351-15364.

61. Vozzi, C.; Negro, M.; Calegari, F.; Stagira, S.; Kovács, K.; Tosa, V. Phase-matching effects in the generation of high-energy photons by mid-infrared few-cycle laser pulses. New J. Phys. 2011, 13, 073003.

62. Chen, M.C.; Arpin, P.; Popmintchev, T.; Gerrity, M.; Zhang, B.; Seaberg, M.; Popmintchev, D.; Murnane, M.; Kapteyn, H. Bright, coherent, ultrafast soft X-ray harmonics spanning the water window from a tabletop light source. Phys. Rev. Lett. 2010, 105, 173901.

63. Popmintchev, T.; Chen, M.C.; Popmintchev, D.; Arpin, P.; Brown, S.; Ališauskas, S.; Andriukaitis, G.; Balčiunas, T.; Mücke, O.D.; Pugzlys, A.; et al. Bright coherent ultrahigh harmonics in the keV X-ray regime from mid-infrared femtosecond lasers. Science 2012, 336, 1287-1291.

64. Cousin, S.; Silva, F.; Teichmann, S.; Hemmer, M.; Buades, B.; Biegert, J. High-flux table-top soft X-ray source driven by sub-2-cycle, CEP stable, $1.85-\mu \mathrm{m}$ 1-kHz pulses for carbon K-edge spectroscopy. Opt. Lett. 2014, $39,5383-5386$.

65. Johnson, A.; Miseikis, L.; Wood, D.; Austin, D.; Brahms, C.; Jarosch, S.; Strüber, C.; Ye, P.; Marangos, J. Measurement of sulfur L2, 3 and carbon K edge XANES in a polythiophene film using a high harmonic supercontinuum. Struct. Dyn. 2016, 3, 062603.

66. Itatani, J.; Levesque, J.; Zeidler, D.; Niikura, H.; Pépin, H.; Kieffer, J.C.; Corkum, P.B.; Villeneuve, D.M. Tomographic imaging of molecular orbitals. Nature 2004, 432, 867-871.

67. Vozzi, C.; Negro, M.; Calegari, F.; Sansone, G.; Nisoli, M.; De Silvestri, S.; Stagira, S. Generalized molecular orbital tomography. Nat. Phys. 2011, 7, 822-826. 
68. Torres, R.; Siegel, T.; Brugnera, L.; Procino, I.; Underwood, J.G.; Altucci, C.; Velotta, R.; Springate, E.; Froud, C.; Turcu, I.; et al. Extension of high harmonic spectroscopy in molecules by a $1300 \mathrm{~nm}$ laser field. Opt. Express 2010, 18, 3174-3180.

69. Vozzi, C.; Torres, R.; Negro, M.; Brugnera, L.; Siegel, T.; Altucci, C.; Velotta, R.; Frassetto, F.; Poletto, L.; Villoresi, P.; et al. High harmonic generation spectroscopy of hydrocarbons. Appl. Phys. Lett. 2010, 97, 241103.

70. Shiner, A.; Schmidt, B.; Trallero-Herrero, C.; Wörner, H.; Patchkovskii, S.; Corkum, P.; Kieffer, J.; Légaré, F.; Villeneuve, D. Probing collective multi-electron dynamics in xenon with high-harmonic spectroscopy. Nat. Phys. 2011, 7, 464-467.

71. Faccialà, D.; Pabst, S.; Bruner, B.D.; Ciriolo, A.G.; De Silvestri, S.; Devetta, M.; Negro, M.; Soifer, H.; Stagira, S.; Dudovich, N.; et al. Probe of Multi-electron Dynamics in Xenon by Caustics in High Order Harmonic Generation. arXiv 2016, arXiv:1603.01755.

72. Pabst, S.; Santra, R. Strong-field many-body physics and the giant enhancement in the high-harmonic spectrum of xenon. Phys. Rev. Lett. 2013, 111, 233005.

73. Worner, H.; Bertrand, J.; Hockett, P.; Corkum, P.; Villeneuve, D. Controlling the interference of multiple molecular orbitals in high-harmonic generation. Phys. Rev. Lett. 2010, 104, 233904.

74. Jin, C.; Bertrand, J.B.; Lucchese, R.; Wörner, H.; Corkum, P.B.; Villeneuve, D.; Le, A.T.; Lin, C.D. Intensity dependence of multiple orbital contributions and shape resonance in high-order harmonic generation of aligned $\mathrm{N}_{2}$ molecules. Phys. Rev. A 2012, 85, 013405.

75. Ghimire, S.; DiChiara, A.D.; Sistrunk, E.; Agostini, P.; DiMauro, L.F.; Reis, D.A. Observation of high-order harmonic generation in a bulk crystal. Nat. Phys. 2011, 7, 138-141.

76. Vampa, G.; Hammond, T.; Thiré, N.; Schmidt, B.; Légaré, F.; McDonald, C.; Brabec, T.; Corkum, P. Linking high harmonics from gases and solids. Nature 2015, 522, 462-464.

77. Luu, T.T.; Garg, M.; Kruchinin, S.Y.; Moulet, A.; Hassan, M.T.; Goulielmakis, E. Extreme ultraviolet high-harmonic spectroscopy of solids. Nature 2015, 521, 498-502.

78. Schubert, O.; Hohenleutner, M.; Langer, F.; Urbanek, B.; Lange, C.; Huttner, U.; Golde, D.; Meier, T.; Kira, M.; Koch, S.W.; et al. Sub-cycle control of terahertz high-harmonic generation by dynamical Bloch oscillations. Nat. Photonics 2014, 8, 119-123.

79. Doumy, G.; Wheeler, J.; Roedig, C.; Chirla, R.; Agostini, P.; DiMauro, L.F. Attosecond Synchronization of High-Order Harmonics from Midinfrared Drivers. Phys. Rev. Lett. 2009, 102, 093002.

80. Sola, I.; Mével, E.; Elouga, L.; Constant, E.; Strelkov, V.; Poletto, L.; Villoresi, P.; Benedetti, E.; Caumes, J.P.; Stagira, S.; et al. Controlling attosecond electron dynamics by phase-stabilized polarization gating. Nat. Phys. 2006, 2, 319-322.

81. Sansone, G.; Benedetti, E.; Calegari, F.; Vozzi, C.; Avaldi, L.; Flammini, R.; Poletto, L.; Villoresi, P.; Altucci, C.; Velotta, R.; et al. Isolated single-cycle attosecond pulses. Science 2006, 314, 443-446.

82. Pfeifer, T.; Gallmann, L.; Abel, M.J.; Nagel, P.M.; Neumark, D.M.; Leone, S.R. Heterodyne mixing of laser fields for temporal gating of high-order harmonic generation. Phys. Rev. Lett. 2006, 97, 163901.

83. Goulielmakis, E.; Schultze, M.; Hofstetter, M.; Yakovlev, V.S.; Gagnon, J.; Uiberacker, M.; Aquila, A.L.; Gullikson, E.; Attwood, D.T.; Kienberger, R.; et al. Single-cycle nonlinear optics. Science 2008, 320, 1614-1617.

84. Saito, N.; Ishii, N.; Kanai, T.; Watanabe, S.; Itatani, J. Attosecond streaking measurement of extreme ultraviolet pulses using a long-wavelength electric field. Sci. Rep. 2016, 6, 35594, doi:10.1038/srep35594.

85. Vozzi, C.; Calegari, F.; Frassetto, F.; Poletto, L.; Sansone, G.; Villoresi, P.; Nisoli, M.; De Silvestri, S.; Stagira, S. Coherent continuum generation above $100 \mathrm{eV}$ driven by an ir parametric source in a two-color scheme. Phys. Rev. A 2009, 79, 033842.

86. Calegari, F.; Vozzi, C.; Negro, M.; Sansone, G.; Frassetto, F.; Poletto, L.; Villoresi, P.; Nisoli, M.; De Silvestri, S.; Stagira, S. Efficient continuum generation exceeding $200 \mathrm{eV}$ by intense ultrashort two-color driver. Opt. Lett. 2009, 34, 3125-3127.

87. Tosa, V.; Altucci, C.; Kovács, K.; Negro, M.; Stagira, S.; Vozzi, C.; Velotta, R. Isolated attosecond pulse generation by Two-Mid-IR laser fields. IEEE J. Sel. Top. Quant. Electron. 2012, 18, 239-247.

88. Negro, M.; Vozzi, C.; Kovacs, K.; Altucci, C.; Velotta, R.; Frassetto, F.; Poletto, L.; Villoresi, P.; De Silvestri, S.; Tosa, V.; et al. Gating of high-order harmonics generated by incommensurate two-color mid-IR laser pulses. Laser Phys. Lett. 2011, 8, 875-879.

89. Vincenti, H.; Quéré, F. Attosecond lighthouses: How to use spatiotemporally coupled light fields to generate isolated attosecond pulses. Phys. Rev. Lett. 2012, 108, 113904. 
90. Wheeler, J.A.; Borot, A.; Monchocé, S.; Vincenti, H.; Ricci, A.; Malvache, A.; Lopez-Martens, R.; Quéré, F. Attosecond lighthouses from plasma mirrors. Nat. Photonics 2012, 6, 829-833.

91. Kim, K.T.; Zhang, C.; Ruchon, T.; Hergott, J.F.; Auguste, T.; Villeneuve, D.; Corkum, P.; Quéré, F. Photonic streaking of attosecond pulse trains. Nat. Photonics 2013, 7, 651-656.

92. Silva, F.; Teichmann, S.M.; Cousin, S.L.; Hemmer, M.; Biegert, J. Spatiotemporal isolation of attosecond soft $\mathrm{X}$-ray pulses in the water window. Nat. Commun. 2015, 6, 6611.

93. Zhang, C.; Vampa, G.; Villeneuve, D.; Corkum, P. Attosecond lighthouse driven by sub-two-cycle, $1.8 \mu \mathrm{m}$ laser pulses. J. Phys. B Atom. Mol. Opt. Phys. 2015, 48, 061001.

94. Hagen, K. Stereochemical applications of gas-phase electron diffraction. Parts A and B edited by I. Hargittai and M. Hargittai. Acta Crystallogr. Sect. B Struct. Sci. 1989, 45, 519-520.

95. Meckel, M.; Comtois, D.; Zeidler, D.; Staudte, A.; Pavičić, D.; Bandulet, H.; Pépin, H.; Kieffer, J.; Dörner, R.; Villeneuve, D.; et al. Laser-induced electron tunneling and diffraction. Science 2008, 320, 1478-1482.

96. Blaga, C.I.; Xu, J.; DiChiara, A.D.; Sistrunk, E.; Zhang, K.; Agostini, P.; Miller, T.A.; DiMauro, L.F.; Lin, C. Imaging ultrafast molecular dynamics with laser-induced electron diffraction. Nature 2012, 483, 194-197.

97. Pullen, M.G.; Wolter, B.; Le, A.T.; Baudisch, M.; Hemmer, M.; Senftleben, A.; Schröter, C.D.; Ullrich, J.; Moshammer, R.; Lin, C.D.; et al. Imaging an aligned polyatomic molecule with laser-induced electron diffraction. Nat. Commun. 2015, 6, 7262.

98. Pullen, M.; Wolter, B.; Le, A.T.; Baudisch, M.; Sclafani, M.; Pires, H.; Schröter, C.; Ullrich, J.; Moshammer, R.; Pfeifer, T.; et al. Influence of orbital symmetry on diffraction imaging with rescattering electron wave packets. Nat. Commun. 2016, 7, 11922.

99. Wolter, B.; Pullen, M.; Le, A.T.; Baudisch, M.; Doblhoff-Dier, K.; Senftleben, A.; Hemmer, M.; Schröter, C.; Ullrich, J.; Pfeifer, T.; et al. Ultrafast electron diffraction imaging of bond breaking in di-ionized acetylene. Science 2016, 354, 308-312.

100. Bian, X.B.; Huismans, Y.; Smirnova, O.; Yuan, K.J.; Vrakking, M.; Bandrauk, A.D. Subcycle interference dynamics of time-resolved photoelectron holography with midinfrared laser pulses. Phys. Rev. A 2011, $84,043420$.

101. Zhou, Y.; Tolstikhin, O.I.; Morishita, T. Near-forward rescattering photoelectron holography in strong-field ionization: Extraction of the phase of the scattering amplitude. Phys. Rev. Lett. 2016, 116, 173001.

102. Huismans, Y.; Rouzée, A.; Gijsbertsen, A.; Jungmann, J.; Smolkowska, A.; Logman, P.; Lepine, F.; Cauchy, C.; Zamith, S.; Marchenko, T.; et al. Time-resolved holography with photoelectrons. Science 2011, 331, 61-64.

103. Meckel, M.; Staudte, A.; Patchkovskii, S.; Villeneuve, D.; Corkum, P.; Dörner, R.; Spanner, M. Signatures of the continuum electron phase in molecular strong-field photoelectron holography. Nat. Phys. 2014, 10,594-600.

104. Huismans, Y.; Gijsbertsen, A.; Smolkowska, A.; Jungmann, J.; Rouzée, A.; Logman, P.; Lepine, F.; Cauchy, C.; Zamith, S.; Marchenko, T.; et al. Scaling laws for photoelectron holography in the midinfrared wavelength regime. Phys. Rev. Lett. 2012, 109, 013002.

105. Herink, G.; Solli, D.; Gulde, M.; Ropers, C. Field-driven photoemission from nanostructures quenches the quiver motion. Nature 2012, 483, 190-193.

106. Teichmann, S.; Rácz, P.; Ciappina, M.F.; Pérez-Hernández, J.; Thai, A.; Fekete, J.; Elezzabi, A.; Veisz, L.; Biegert, J.; Dombi, P. Strong-field plasmonic photoemission in the mid-IR at $<1 \mathrm{GW} / \mathrm{cm} 2$ intensity. Sci. Rep. 2015, 5, doi:10.1038/srep07584.

107. McNeil, B.; Thompson, N.; Dunning, D.; Sheehy, B. High harmonic attosecond pulse train amplification in a free electron laser. J. Phys. B Atom. Mol. Opt. Phys. 2011, 44, 065404.

108. Austin, D.R.; Kafka, K.R.; Lai, Y.H.; Wang, Z.; Zhang, K.; Li, H.; Blaga, C.I.; Allen, Y.Y.; DiMauro, L.F.; Chowdhury, E.A. High spatial frequency laser induced periodic surface structure formation in germanium by mid-IR femtosecond pulses. J. Appl. Phys. 2016, 120, 143103.

109. Konig, K.; Riemann, I.; Fischer, P.; Halbhuber, K. In tracellular nanosurgery with near infrared femtosecond laser pulses. Cell. Mol. Biol. 1999, 45, 195-201.

(C) 2017 by the authors. Licensee MDPI, Basel, Switzerland. This article is an open access article distributed under the terms and conditions of the Creative Commons Attribution (CC BY) license (http:/ / creativecommons.org/licenses/by/4.0/). 